\title{
NUMERICAL APPROXIMATION OF A NON-SMOOTH PHASE-FIELD MODEL FOR MULTICOMPONENT INCOMPRESSIBLE FLOW
}

\author{
LUUBOMÍR BAŇAS AND ROBERT NÜRNBERG
}

\begin{abstract}
We present a phase-field model for multiphase flow for an arbitrary number of immiscible incompressible fluids with variable densities and viscosities. The model consists of a system of the Navier-Stokes equations coupled to multicomponent Cahn-Hilliard variational inequalities. The proposed formulation admits a natural energy law, preserves physically meaningful constraints and allows for a straightforward modelling of surface tension effects. We propose a practical fully discrete finite element approximation of the model which preserves the energy law and the associated physical constraints. In the case of matched densities we prove convergence of the numerical scheme towards a weak solution of the continuous model. The convergence of the numerical approximations also implies the existence of weak solutions. Furthermore, we propose a convergent iterative fixed-point algorithm for the solution of the discrete nonlinear system of equations and present several computational studies of the proposed model.
\end{abstract}

\section{INTRODUCTION}

Let $\Omega$ be a bounded domain in $\mathbb{R}^{d}, d=2,3$. We consider a mixture of $N \geq 2$ immiscible incompressible fluids and introduce a vector valued order parameter $\mathbf{c}=\left(c_{1}, c_{2}, \ldots, c_{N}\right)^{T}$ : $\Omega \rightarrow \mathbb{R}^{N}$, where $0 \leq c_{i} \leq 1, i=1, \ldots, N$ are order parameters corresponding to the different fluid components. Physically meaningful values for the order parameter $\mathbf{c}$ have nonnegative entries and satisfy $\sum_{n=1}^{N} c_{n}=1$. It is therefore convenient to define the Gibbs simplex

$$
\mathbb{G}^{N}=\left\{\boldsymbol{\zeta} \in \mathbb{R}^{N}: \sum_{n=1}^{N} \zeta_{n}=1, \boldsymbol{\zeta} \geq \mathbf{0}\right\} \subset \mathbb{R}^{N}
$$

as the set of meaningful values for c, i.e. $\mathbf{c} \in \mathbb{G}^{N}$. For later use, we denote the corners of the Gibbs simplex by $\boldsymbol{e}_{i}, i=1, \ldots, N$. Similarly we define the vector valued chemical potential as $\mathbf{w}=\left(w_{1}, w_{2}, \ldots, w_{N}\right)^{T} \in \mathbb{R}^{N}$. In addition, we let $\underline{\mathbf{u}}: \Omega \rightarrow \mathbb{R}^{d}$ and $p: \Omega \rightarrow \mathbb{R}$ denote the velocity and pressure of the fluid mixture, with its density and viscosity defined by $\rho(\mathbf{c})=\mathbf{c}^{T} \boldsymbol{\rho}$ and $\mu(\mathbf{c})=\mathbf{c}^{T} \boldsymbol{\mu}$, respectively. Here $\boldsymbol{\rho}=\left(\rho_{1}, \rho_{2}, \ldots, \rho_{N}\right)^{T} \in \mathbb{R}^{N}$ and $\boldsymbol{\mu}=\left(\mu_{1}, \mu_{2}, \ldots, \mu_{N}\right)^{T} \in$ $\mathbb{R}^{N}$ denote the densities and viscosities of the individual fluid components, which satisfy $\rho_{\min } \leq \rho_{i} \leq \rho_{\max }, \mu_{\min } \leq \mu_{i} \leq \mu_{\min }$ for $i=1, \ldots, N$, with $\rho_{\min }, \rho_{\max }, \mu_{\min }, \mu_{\max } \in \mathbb{R}_{>0}$. We propose a phase-field model for a mixture of $N$ incompressible immiscible fluids. The model consists of a system of variable density multicomponent Cahn-Hilliard-Navier-Stokes 
equations

$$
\partial_{t}(\rho(\mathbf{c}) \underline{\mathbf{u}})+\nabla \cdot(\rho(\mathbf{c}) \underline{\mathbf{u}} \otimes \underline{\mathbf{u}})+\nabla \cdot(\underline{\mathbf{u}} \otimes \underline{\mathbf{j}})-\nabla \cdot(2 \mu(\mathbf{c}) D(\underline{\mathbf{u}}))+\nabla p=\rho(\mathbf{c}) \underline{\mathbf{g}}-\lambda(\nabla \mathbf{w})^{T} \mathbf{c},
$$

$$
\begin{aligned}
\nabla \cdot \underline{\mathbf{u}} & =0, \\
\partial_{t} \mathbf{c}+\nabla \cdot(\mathbf{c} \otimes \underline{\mathbf{u}}) & =\nabla \cdot(M \nabla \mathbf{w}), \\
\mathbf{w} & =-\varepsilon \Delta \mathbf{c}+\frac{1}{\varepsilon} \frac{\partial \Psi(\mathbf{c})}{\partial \mathbf{c}},
\end{aligned}
$$

where $D(\underline{\mathbf{u}})=\frac{1}{2}\left(\nabla \underline{\mathbf{u}}+(\nabla \underline{\mathbf{u}})^{T}\right)$ denotes the rate-of-deformation tensor and $\nabla \boldsymbol{\eta}$ is the $N \times d$ matrix with entries $\left(\frac{\partial \eta_{m}}{\partial x_{p}}\right)_{m=1, \ldots, N, p=1, \ldots, d}$, for $\boldsymbol{\eta} \in \mathbb{R}^{N}$. For a $N \times d$ matrix $\Lambda=\left(\Lambda_{m p}\right)_{m=1, \ldots, N, p=1, \ldots, d}$, $\nabla \cdot \Lambda$ is the $N \times 1$ vector with entries $\sum_{p=1}^{d} \frac{\partial \Lambda_{m p}}{\partial x_{p}}, m=1, \ldots, N$. The mobility matrix $M=\left(m_{i j}\right)_{i, j=1, \ldots, N} \in \mathbb{R}^{N \times N}$ is described in more detail below. Moreover, we define

$$
\underline{\mathbf{j}}=-\sum_{i=1}^{N} \rho_{i} \sum_{j=1}^{N} m_{i j} \nabla w_{j}=-(M \nabla \mathbf{w})^{T} \boldsymbol{\rho} .
$$

We consider homogeneous Neumann and Dirichlet boundary conditions for the Cahn-Hilliard variables $\mathbf{c}, \mathbf{w}$ and the velocity field $\underline{\mathbf{u}}$, respectively, i.e.

$$
\frac{\partial \mathbf{c}}{\partial \underline{\mathbf{n}}}=M \frac{\partial \mathbf{w}}{\partial \underline{\mathbf{n}}}=\mathbf{0} \quad \text { and } \quad \underline{\mathbf{u}}=\underline{\mathbf{0}} \quad \text { on } \quad(0, T) \times \partial \Omega,
$$

where $\underline{\mathbf{n}}$ is the outward unit normal vector to $\partial \Omega$. In addition, $\underline{\mathbf{u}}$ and $\mathbf{c}$ satisfy the initial conditions

$$
\underline{\mathbf{u}}(0, \underline{\mathbf{x}})=\underline{\mathbf{u}}^{0}(\underline{\mathbf{x}}) \quad \forall \underline{\mathbf{x}} \in \Omega \quad \text { and } \quad \mathbf{c}(0, \underline{\mathbf{x}})=c^{0}(\underline{\mathbf{x}}) \quad \forall \underline{\mathbf{x}} \in \Omega .
$$

Here we assume the following properties for the initial condition $\mathbf{c}^{0}$ for the variable $\mathbf{c}$

$$
\text { (a) } \quad \mathbf{c}^{0}(\underline{\mathbf{x}}) \geq \mathbf{0} \quad \text { and } \quad(b) \quad \sum_{i=1}^{N} c_{i}^{0}(\underline{\mathbf{x}})=1 \quad \forall \underline{\mathbf{x}} \in \Omega \text {, }
$$

i.e. that $\mathbf{c}^{0}(\underline{\mathbf{x}}) \in \mathbb{G}^{N}$ for all $\underline{\mathbf{x}} \in \Omega$.

The Ginzburg-Landau free energy of the Cahn-Hilliard part of the system takes the form

$$
\mathcal{E}_{c h}(\mathbf{c})=\int_{\Omega}\left(\frac{\varepsilon}{2}|\nabla \mathbf{c}|^{2}+\frac{1}{\varepsilon} \Psi(\mathbf{c})\right),
$$

where the homogeneous free energy is expressed as

$$
\Psi(\mathbf{c})=\Psi_{0}(\mathbf{c})-\frac{1}{2} \mathbf{c}^{T} A \mathbf{c},
$$

with $A$ a constant symmetric $N \times N$ matrix that models the surface tension forces between the different fluids. Here a physically reasonable assumption is that $\operatorname{diag}(A)=0$ and $A_{i j}<0$, for $i \neq j$. For equal surface tension forces, which leads to equal $120^{\circ}$ angle conditions at triple junctions, the matrix $A$ in (1.7) takes the form

$$
A=I-11^{T}
$$

where $I$ is the $N \times N$ identity matrix and $\mathbf{1}=(1, \ldots, 1)^{T} \in \mathbb{R}^{N}$. The choice of $A$ for more general contact angle conditions has been discussed in e.g. [15, 29], see also Sections 2.4 and 5.2 below. 
In this paper the term $\Psi_{0}$ in (1.7) represents the obstacle free energy

$$
\Psi_{0}(\mathbf{c})= \begin{cases}0 & \text { if } \mathbf{c} \in \mathbb{G}^{N}, \\ \infty & \text { if } \mathbf{c} \notin \mathbb{G}^{N} .\end{cases}
$$

For the non-smooth free energy (1.7) the derivative $\frac{\partial \Psi(\mathbf{c})}{\partial \mathbf{c}}=\left(\partial_{c_{1}} \Psi(\mathbf{c}), \ldots, \partial_{c_{N}} \Psi(\mathbf{c})\right)^{T}$ is only formal and the equation (1.1d) has to be formulated as a variational inequality, cf. [29]. The mobility matrix $M$ is symmetric positive semi-definite, with the natural property

$$
M 1=0 .
$$

This property ensures that the constraint $\sum_{i=1}^{N} c_{i}=1$ is fulfilled during the evolution, as long as the initial data satisfies (1.5b). Moreover, for the constant density case it follows from (1.2) and (1.10) that $\mathbf{j}$ vanishes, meaning that our model is consistent with classical models for twoand multi-phase flow. For instance, in the case of a constant mobility $m_{0} \in \mathbb{R}_{>0}$, the mobility matrix $M=\left(m_{i j}\right)_{i j=1}^{N}$ is defined by the entries

$$
m_{i j}= \begin{cases}m_{0}(1-1 / N) & \text { if } i=j \\ -m_{0} / N & \text { if } i \neq j\end{cases}
$$

An alternative option is a concentration dependent mobility matrix $M(\mathbf{c})$ with entries

$$
m_{i j}(\mathbf{c})=m_{0}\left(c_{i}+\nu\right)\left(\delta_{i j}-\frac{c_{j}+\nu}{1+N \nu}\right),
$$

where $\nu>0$ is a fixed parameter. Note, that for $\nu \rightarrow 0$ in (1.12), we recover a degenerate concentration dependent mobility matrix

$$
m_{i j}(\mathbf{c})=m_{0} c_{i}\left(\delta_{i j}-c_{j}\right)
$$

cf. [7, 29]. Other choices for the mobility matrix are also possible, see e.g. [15, 7, 29].

For simplicity we will consider the constant mobility (1.11) throughout the theoretical part of this paper. However, it is straightforward to generalize the presented numerical approximations to the case of degenerate and concentration dependent mobilities, such as, e.g., (1.13). In particular, all theoretical results from this paper remain valid for the model with the regularized variant (1.12) of the degenerate mobility (1.13). Furthermore, Lemmas 2, 3, 5 remain valid for the numerical approximation with degenerate mobility (1.13), however, the existence of discrete solutions is not clear, cf. [7].

Energy preserving convergent numerical approximations of variable density Navier-Stokes equations have been constructed for instance in [27, 2]. In principle, the considered so-called "sharp interface" model allows an arbitrary number of components to be included. However, the corresponding numerical approximations suffer from numerical diffusion. Furthermore, the inclusion of surface tension effects in variable density Navier-Stokes equations is not straightforward. Phase-field models have the advantage that various physical effects, such as surface tension, can be included in a straightforward way. Furthermore, the corresponding numerical approximations do not suffer from numerical diffusion, and preserve properties of the continuous models, such as mass conservation and energy estimates. Phase-field models for binary fluid mixtures and their numerical approximations are well-studied. Convergence of numerical approximations for density independent models of two-phase flows has been shown in $[16,21]$. A thermodynamically consistent phase-field model for two-phase flows has been proposed in [1]; energy preserving numerical approximations for that model have been considered in [20,17], while convergence of a numerical approximation is shown in [18], [19]. 
Considerably fewer results are available for mixtures of more than two fluids. For different approaches to construct phase-field models for ternary fluids with smooth homogeneous free energy, we refer to [25, 9], the review paper [24] and the references therein. Stable numerical approximations for density dependent three-phase flows have been proposed in [10, 28], and convergence of these numerical approximations towards weak solutions has been shown in the case of constant density. Development of phase-field models for $N>3$ fluid phases is not straightforward due to the complicated modelling of surface tension effects, cf. [23, 26]. Recently, generalizations of the thermodynamically consistent phase-field model from [1] for variable density flows with an arbitrary number of phases and their numerical approximations have been proposed in [13, 14]. We also mention the work [11], which proposes consistent $N$-component Cahn-Hilliard models with smooth free energy, as well as their numerical approximation and the coupling with Navier-Stokes equations. For the numerical approximation of multicomponent Cahn-Hilliard systems in the absence of fluid flow, including the case of degenerate mobility (1.13) and for the non-smooth obstacle free energy (1.7), see e.g. [7, 29]. In the present work we propose a generalization of the thermodynamically consistent model from [1] for an arbitrary number of fluid components. As far as we are aware, all existing phase-field models for fluid flow use a smooth or regularized free energy formulation and the present model is the first one to employ a non-smooth free energy. The advantage of the nonsmooth obstacle energy (1.9) is that the pure phases are easily identified, and that physically meaningful values for $\mathbf{c}$ are guaranteed throughout. We construct an energy stable numerical approximation of the model and show convergence of the approximation to a weak solution in the case of fluids with equal densities. Apart from the present paper, convergence of numerical approximations of Cahn-Hilliard-Navier-Stokes systems for $N>2$ components has only been show in $[10,28]$. We note that their results are limited to $N=3$ components, while the results presented in this paper hold for arbitrary $N \geq 2$. In addition, the convergence of our numerical approximations implies the existence of weak solutions to (1.1) for a non-smooth energy, which is a new result even for $N=2$. Furthermore, as detailed in Remark 1, the existence of discrete solutions requires a velocity-pressure-density compatibility condition for the corresponding finite element spaces to hold ${ }^{1}$.

The remainder of the paper is organized as follows. In Section 2 we discuss properties of the continuous model, such as the energy inequality, surface tension effects and consistency with models for $N=2$ fluid components. The density independent model is considered in detail in Section 3. Here we propose a fully discrete energy preserving numerical approximation of the model and prove convergence towards a weak solution. Furthermore, we present a simple fixed point algorithm for the solution of the discrete nonlinear system of equations and show its convergence. In Section 4 we construct an energy preserving numerical approximation of the density dependent model. The paper concludes with Section 5, where we briefly discuss implementation issues, such as mesh adaptivity and algebraic solvers, and where we present numerical studies of the model.

\section{Properties of The MOdeL}

Below we summarize the main properties of the model (1.1).

\footnotetext{
${ }^{1}$ Note, that an analogical velocity-pressure-density compatibility condition was used previously in [27, 2] where the former used a piecewise constant pressure-density spaces, and the latter used piecewise linear continuous pressure-density spaces.
} 
2.1. Energy law. An energy law for the system (1.1) can be obtained by the following formal calculations. Using the representation for the density $\rho(\mathbf{c})=\mathbf{c}^{T} \boldsymbol{\rho}$ and (1.2) we obtain from (1.1c) that

$$
\partial_{t} \rho(\mathbf{c})+\nabla \cdot(\rho(\mathbf{c}) \underline{\mathbf{u}})+\nabla \cdot \underline{\mathbf{j}}=0 .
$$

We multiply (2.1) by $|\underline{\mathbf{u}}|^{2}$, integrate over $\Omega$ and use integration by parts in the second and third terms to get

$$
\begin{aligned}
\int_{\Omega} \partial_{t} \rho(\mathbf{c})|\underline{\mathbf{u}}|^{2}+\int_{\Omega}[\nabla \cdot(\rho(\mathbf{c}) \underline{\mathbf{u}})]|\underline{\mathbf{u}}|^{2}+\int_{\Omega}[\nabla \cdot \underline{\mathbf{j}}]|\underline{\mathbf{u}}|^{2} \\
=\int_{\Omega} \partial_{t} \rho(\mathbf{c})|\underline{\mathbf{u}}|^{2}-2 \int_{\Omega} \rho(\mathbf{c})(\underline{\mathbf{u}} \cdot \nabla) \underline{\mathbf{u}} \cdot \underline{\mathbf{u}}-2 \int_{\Omega}(\underline{\mathbf{j}} \cdot \nabla) \underline{\mathbf{u}} \cdot \underline{\mathbf{u}}=0 .
\end{aligned}
$$

Next, we take a product of $(1.1 \mathrm{a}, \mathrm{c}, \mathrm{d})$ with $\underline{\mathbf{u}}, \mathbf{w}, \partial_{t} \mathbf{c}$, respectively, use $(1.1 \mathrm{~b})$ and integrate over $\Omega$ and by parts to yield

$$
\begin{aligned}
& \frac{1}{2} \partial_{t} \int_{\Omega} \rho(\mathbf{c})|\underline{\mathbf{u}}|^{2}+\int_{\Omega} 2 \mu(\mathbf{c})|D(\underline{\mathbf{u}})|^{2}+\frac{1}{2} \int_{\Omega} \partial_{t} \rho(\mathbf{c})|\underline{\mathbf{u}}|^{2} \\
& \quad-\int_{\Omega} \rho(\mathbf{c})(\underline{\mathbf{u}} \cdot \nabla) \underline{\mathbf{u}} \cdot \underline{\mathbf{u}}-\int_{\Omega}(\underline{\mathbf{j}} \cdot \nabla) \underline{\mathbf{u}} \cdot \underline{\mathbf{u}}=\int_{\Omega} \rho(\mathbf{c}) \underline{\mathbf{g}} \cdot \underline{\mathbf{u}}-\lambda \int_{\Omega}(\nabla \mathbf{w})^{T} \mathbf{c} \cdot \underline{\mathbf{u}}, \\
& \int_{\Omega} M \nabla \mathbf{w} \cdot \nabla \mathbf{w}=-\int_{\Omega} \partial_{t} \mathbf{c} \cdot \mathbf{w}+\int_{\Omega}(\nabla \mathbf{w})^{T} \mathbf{c} \cdot \underline{\mathbf{u}}, \\
& \frac{\varepsilon}{2} \partial_{t} \int_{\Omega}|\nabla \mathbf{c}|^{2}+\frac{1}{\varepsilon} \partial_{t} \int_{\Omega} \Psi(\mathbf{c})=\left(\mathbf{w}, \partial_{t} \mathbf{c}\right) .
\end{aligned}
$$

Now we use (2.2), multiply (2.3b) and (2.3c) by $\lambda$ to cancel out the terms on the right-hand side, and sum the equations up to get the energy identity

$$
\lambda\left[\partial_{t} \mathcal{E}_{c h}(\mathbf{c})+\int_{\Omega}\left|M^{\frac{1}{2}} \nabla \mathbf{w}\right|^{2}\right]+\partial_{t} \mathcal{E}_{n s}(\mathbf{c}, \underline{\mathbf{u}})+\int_{\Omega} 2 \mu(\mathbf{c})|D(\underline{\mathbf{u}})|^{2}=\int_{\Omega} \rho(\mathbf{c}) \underline{\mathbf{g}} \cdot \underline{\mathbf{u}},
$$

where

$$
\mathcal{E}_{n s}(\mathbf{c}, \underline{\mathbf{u}})=\frac{1}{2} \int_{\Omega} \rho(\mathbf{c})|\underline{\mathbf{u}}|^{2}
$$

is the kinetic energy of the Navier-Stokes part of the system (1.1).

The identity (2.2) was necessary for the derivation of the energy law (2.4). However, on the discrete level (2.1) does not hold in general. Hence, a discrete energy estimate is not immediately obvious. To derive an energy preserving numerical approximation, in Section 4 we consider an equivalent reformulation of (1.1). On noting $\nabla \cdot(\underline{\mathbf{u}} \otimes \underline{\mathbf{j}})=(\underline{\mathbf{j}} \cdot \nabla) \underline{\mathbf{u}}+(\nabla \cdot \underline{\mathbf{j}}) \underline{\mathbf{u}}$ we obtain by a direct calculation that

$$
\begin{aligned}
\partial_{t}(\rho(\mathbf{c}) \underline{\mathbf{u}})+\nabla \cdot(\rho(\mathbf{c}) \underline{\mathbf{u}} \otimes \underline{\mathbf{u}})+\nabla \cdot(\underline{\mathbf{u}} \otimes \underline{\mathbf{j}})= & \rho(\mathbf{c}) \partial_{t} \underline{\mathbf{u}}+(\rho(\mathbf{c}) \underline{\mathbf{u}} \cdot \nabla) \underline{\mathbf{u}}+(\underline{\mathbf{j}} \cdot \nabla) \underline{\mathbf{u}} \\
& +\left[\partial_{t} \rho(\mathbf{c})+\nabla \cdot(\rho(\mathbf{c}) \underline{\mathbf{u}})+\nabla \cdot \underline{\mathbf{j}}\right] \underline{\mathbf{u}},
\end{aligned}
$$

which implies that

$$
\begin{aligned}
\frac{1}{2}\left[\partial_{t} \rho(\mathbf{c})+\right. & \nabla \cdot(\rho(\mathbf{c}) \underline{\mathbf{u}})+\nabla \cdot \underline{\mathbf{j}}] \underline{\mathbf{u}} \\
& =\frac{1}{2}\left\{\partial_{t}(\rho(\mathbf{c}) \underline{\mathbf{u}})-\nabla \cdot(\underline{\mathbf{u}} \otimes[\rho(\mathbf{c}) \underline{\mathbf{u}}+\underline{\mathbf{j}}])-\rho(\mathbf{c}) \partial_{t} \underline{\mathbf{u}}+([\rho(\mathbf{c}) \underline{\mathbf{u}}+\underline{\mathbf{j}}] \cdot \nabla) \underline{\mathbf{u}}\right\} .
\end{aligned}
$$


The identities (2.5) and (2.1) then allow the momentum equation to be reformulated as follows

$$
\begin{aligned}
& \partial_{t}(\rho(\mathbf{c}) \underline{\mathbf{u}})+\nabla \cdot(\rho(\mathbf{c}) \underline{\mathbf{u}} \otimes \underline{\mathbf{u}})+\nabla \cdot(\underline{\mathbf{u}} \otimes \underline{\mathbf{j}})-\underbrace{\frac{1}{2}\left[\partial_{t} \rho(\mathbf{c})+\nabla \cdot(\rho(\mathbf{c}) \underline{\mathbf{u}})+\nabla \cdot \underline{\mathbf{j}}\right] \underline{\mathbf{u}}}_{=0} \\
&=\partial_{t}(\rho(\mathbf{c}) \underline{\mathbf{u}})+\nabla \cdot(\rho(\mathbf{c}) \underline{\mathbf{u}} \otimes \underline{\mathbf{u}})+\nabla \cdot(\underline{\mathbf{u}} \otimes \underline{\mathbf{j}}) \\
&-\frac{1}{2}\left\{\partial_{t}(\rho(\mathbf{c}) \underline{\mathbf{u}})+\nabla \cdot(\underline{\mathbf{u}} \otimes[\rho(\mathbf{c}) \underline{\mathbf{u}}+\underline{\mathbf{j}}])-\rho(\mathbf{c}) \partial_{t} \underline{\mathbf{u}}-([\rho(\mathbf{c}) \underline{\mathbf{u}}+\underline{\mathbf{j}}] \cdot \nabla) \underline{\mathbf{u}}\right\} \\
&= \frac{1}{2}\left\{\partial_{t}(\rho(\mathbf{c}) \underline{\mathbf{u}})+\nabla \cdot(\rho(\mathbf{c}) \underline{\mathbf{u}} \otimes \underline{\mathbf{u}})+\nabla \cdot(\underline{\mathbf{u}} \otimes \underline{\mathbf{j}})+\rho(\mathbf{c}) \partial_{t} \underline{\mathbf{u}}+(\rho(\mathbf{c}) \underline{\mathbf{u}} \cdot \nabla) \underline{\mathbf{u}}+(\underline{\mathbf{j}} \cdot \nabla) \underline{\mathbf{u}}\right\} .
\end{aligned}
$$

By (2.6) the momentum equation (1.1a) is equivalent to

$$
\begin{gathered}
\frac{1}{2}\left\{\partial_{t}(\rho(\mathbf{c}) \underline{\mathbf{u}})+\rho(\mathbf{c}) \partial_{t} \underline{\mathbf{u}}+\nabla \cdot(\rho(\mathbf{c}) \underline{\mathbf{u}} \otimes \underline{\mathbf{u}})+(\rho(\mathbf{c}) \underline{\mathbf{u}} \cdot \nabla) \underline{\mathbf{u}}+\nabla \cdot(\underline{\mathbf{u}} \otimes \underline{\mathbf{j}})+(\underline{\mathbf{j}} \cdot \nabla) \underline{\mathbf{u}}\right\} \\
=\rho(\mathbf{c}) \underline{\mathbf{g}}-\lambda(\nabla \mathbf{w})^{T} \mathbf{c} .
\end{gathered}
$$

Hence, the energy law (2.4) for the reformulated system (2.7), (1.1b)-(1.1d) then follows analogously as before, with the exception that (2.2) is not used in the calculations, cf. Lemma 5 below.

2.2. Conservation properties. Next, we state conservation properties of the model which are due to the Cahn-Hilliard part of (1.1). From (1.5) it follows, cf. [5, 6], that the solution of (1.1) satisfies

$$
\text { (a) } \mathbf{c}(t, \underline{\mathbf{x}}) \geq \mathbf{0} \quad \text { and } \quad(b) \quad \sum_{i=1}^{N} c_{i}(t, \underline{\mathbf{x}})=1 \quad \forall \underline{\mathbf{x}} \in \Omega, t \in[0, T],
$$

where $(2.8 \mathrm{a}, \mathrm{b})$ are enforced by the homogeneous free energy, since (1.9) becomes infinite for c $\notin \mathbb{G}^{N}$. The condition $(2.8 \mathrm{~b})$ is also a consequence of (1.1c) and the incompressibility condition (1.1b). Namely, using (1.10) and $\nabla \cdot \underline{\mathbf{u}}=0$ we obtain from (1.1c) that

$$
\partial_{t}\left(\mathbf{1}^{T} \mathbf{c}\right)+[\underline{\mathbf{u}} \cdot \nabla]\left(\mathbf{1}^{T} \mathbf{c}\right)=0,
$$

and so (1.5b) yields that $\mathbf{1}^{T} \mathbf{c}=1$ holds for all times.

The boundary conditions imply that the mass of the individual components is preserved, i.e.,

$$
\int_{\Omega} \mathbf{c}(t, \cdot)=\int_{\Omega} \mathbf{c}^{0} \quad \forall t \in[0, T]
$$

Note that because of the conservative form of the convective term in (1.1c), the incompressibility condition $\nabla \cdot \underline{\mathbf{u}}=0$ is not required for the global mass conservation (2.9) to hold.

2.3. Consistency with binary phase-field models. In the case $N=2$, assuming that $A$ is of the form (1.8) and that $M$ is of the form (1.11), we define $c=c_{2}-c_{1}$ and $w=w_{2}-w_{1}$, 
cf. $[8,4]$. We then obtain that $(\underline{\mathbf{u}}, p, c, w)$ satisfy the following set of equations.

$$
\partial_{t}(\tilde{\rho}(c) \underline{\mathbf{u}})+\nabla \cdot(\tilde{\rho}(c) \underline{\mathbf{u}} \otimes \underline{\mathbf{u}})+\nabla \cdot(\underline{\mathbf{u}} \otimes \underline{\mathbf{j}})-\nabla \cdot(2 \tilde{\mu}(c) D(\underline{\mathbf{u}}))+\nabla p=\tilde{\rho}(c) \underline{\mathbf{g}}-\frac{1}{2} \lambda c \nabla w,
$$

where $\tilde{\rho}(c)=\frac{1-c}{2} \rho_{1}+\frac{1+c}{2} \rho_{2}$, and similarly for $\tilde{\mu}(c)$. Moreover,

$$
\tilde{\Psi}(c)= \begin{cases}\frac{1}{2}\left(1-c^{2}\right) & \text { if }|c| \leq 1, \\ \infty & \text { if }|c|>1,\end{cases}
$$

is the standard obstacle potential. In deriving (2.10a) we have noted that for $|c|<1$ it follows from (1.1d) that $\nabla w_{1}+\nabla w_{2}=0$, and so we obtain that

$$
(\nabla \mathbf{w})^{T} \mathbf{c}=c_{1} \nabla w_{1}+c_{2} \nabla w_{2}=c_{1} \nabla w_{1}-c_{2} \nabla w_{1}=-c \nabla w_{1}=c \frac{1}{2}\left(\nabla w_{2}-\nabla w_{1}\right)=\frac{1}{2} c \nabla w .
$$

Similarly, on noting $M_{i i}=\frac{1}{2}$, we get $\underline{\mathbf{j}}=-\frac{m_{0}}{2}\left(\rho_{2}-\rho_{1}\right) \nabla w$, which corresponds to the model developed in [1] for the non-smooth free energy $\tilde{\Psi}(c)$. Note that so far existence of solutions for the model from [1] has only been shown in the case of a smooth free energy. Our convergence result, on the other hand, covers the non-smooth case for a model with constant density $(\underline{\mathbf{j}}=\underline{\mathbf{0}})$.

2.4. Surface tension. The term $\lambda(\nabla \mathbf{w})^{T} \mathbf{c}=\lambda \sum_{i=1}^{N} c_{i} \nabla w_{i}$ in (1.1a) models capillary forces, i.e., it introduces pressure jumps across the interfaces between the different fluid components. In particular, the pressure jump induced at the interface between the components $i$ and $j$ is proportional to the curvature of the interface and to the surface tension coefficient $\sigma_{i j}$. The coefficients $\sigma_{i j}$ are determined by the matrix $A=\left(A_{i j}\right)_{i, j=1, \ldots, N}$, through the minimization problem

$$
\sigma_{i j}=2 \inf _{\boldsymbol{q}} \int_{-1}^{1}\left|\boldsymbol{q}^{\prime}(s)\right| \sqrt{\frac{1}{2} \Psi(\boldsymbol{q}(s))} \mathrm{d} s, \quad i, j \in\{1, \ldots, N\},
$$

where we recall (1.7) and where the infimum is over all $\boldsymbol{q} \in C^{1}\left([-1,1], \mathbb{R}^{N}\right)$ with $\boldsymbol{q}(-1)=\boldsymbol{e}_{i}$ and $\boldsymbol{q}(1)=\boldsymbol{e}_{j}$; see [29, Eq. (1.13)]. In [22] it is shown that $\lambda(\nabla \mathbf{w})^{T} \mathbf{c}$, for $N=2$, relates to the continuous surface tension force formulation of the capillary force term, where the parameter $\lambda$ is determined by the equilibrium profile of the interface. In [23] a slightly more general formulation $\sum_{i=1}^{N} \lambda_{i} c_{i} \nabla w_{i}$ with parameters $\lambda_{i}, i=1, \ldots, N$, is considered. The author then shows that this choice can only be considered in the case $N \leq 3$ for their CHNS model. This is due to the fact that the determination of the parameters $\lambda_{i}$ in their model, in order to model the correct surface tension coefficients $\sigma_{i j}$, leads to an overdetermined system of equations in the case $N \geq 4$, which has no solution in general.

The advantage of our model (1.1) is that the surface tension can be modelled by $\lambda(\nabla \mathbf{w})^{T} \mathbf{c}$ with a single scaling parameter $\lambda$. To see this, we formally consider an interface between the fluid components $i$ and $j$, so that in the interfacial region $c_{i}, c_{j} \in(0,1)$ and $c_{l}=0$ for all 
$l \in\{1, \ldots, N\} \backslash\{i, j\}$. Hence the Cahn-Hilliard part in (1.1) yields for the chemical potentials that (recall that we assume $A_{i i}=0$ and $A_{i j}<0$, for $i \neq j$ )

$$
w_{i}=-\varepsilon \Delta c_{i}-\frac{\left|A_{i j}\right|}{\varepsilon} c_{j} \quad \text { and } \quad w_{j}=-\varepsilon \Delta c_{j}-\frac{\left|A_{i j}\right|}{\varepsilon} c_{i} .
$$

Setting $c=c_{i}-c_{j} \in(-1,1), w=w_{i}-w_{j}$ we obtain that

$$
w=-\varepsilon \Delta c-\frac{\left|A_{i j}\right|}{\varepsilon} c .
$$

Hence, the Cahn-Hilliard part of (1.1) reduces to the Cahn-Hilliard equation for two component mixtures with interfacial parameter $\tilde{\varepsilon}=\frac{\varepsilon}{\sqrt{\left|A_{i j}\right|}}$. Furthermore, since $c_{i}=1-c_{j}$ and $w_{i}=$ $-\frac{\left|A_{i j}\right|}{\varepsilon}-w_{j}$, we obtain by taking a gradient of the sum of the two equations in (2.13) that $\nabla w_{i}=-\nabla w_{j}$. As in the previous section we obtain that $c_{i} \nabla w_{i}+c_{j} \nabla w_{j}=\frac{1}{2}(c \nabla w)$. Then we can rewrite the capillary term across the interface $\Gamma_{i j}$, using the scaling of [22], as

$$
\frac{\lambda}{2} c \nabla w=\frac{\lambda}{2} \sqrt{\left|A_{i j}\right|} \frac{1}{\tilde{\varepsilon}} c \nabla \tilde{w} .
$$

The above corresponds to a capillary force with surface tension coefficient $\frac{\lambda}{2} \sqrt{\left|A_{i j}\right|}$, where the scaling constant $\lambda$ has to be determined from the equilibrium profile, in order to match the surface tension coefficient $\sigma_{i j}$ in the sharp interface limit, cf. [22].

The above considerations show that our model with capillary term $\lambda(\nabla \mathbf{w})^{T} \mathbf{c}$ can reproduce correct capillary forces and surface tension effects for any number of components. The formulation (1.1) does not need to be modified for $N \geq 4$, which is not the case for the multicomponent model considered in [23]. A potential disadvantage of our model (1.1) is that the widths of the diffuse interfaces are proportional to the surface tension coefficients $\sigma_{i j}$, which leads to different interface widths in the case of unequal surface tensions.

\section{Density independent Model}

In the case of fluids with constant density $\rho(\mathbf{c}) \equiv 1$, i.e. when $\boldsymbol{\rho}=\mathbf{1} \in \mathbb{R}^{N}$, the model (1.1) reduces to

$$
\begin{aligned}
\partial_{t} \underline{\mathbf{u}}+(\underline{\mathbf{u}} \cdot \nabla) \underline{\mathbf{u}}-\nabla \cdot(2 \mu(\mathbf{c}) D(\underline{\mathbf{u}}))+\nabla p & =\underline{\mathbf{g}}-\lambda(\nabla \mathbf{w})^{T} \mathbf{c}, \\
\nabla \cdot \underline{\mathbf{u}} & =0 \\
\partial_{t} \mathbf{c}+\nabla \cdot(\mathbf{c} \otimes \underline{\mathbf{u}}) & =\nabla \cdot(M \nabla \mathbf{w}), \\
\mathbf{w} & =-\varepsilon \Delta \mathbf{c}+\frac{1}{\varepsilon} \frac{\partial \tilde{\Psi}(\mathbf{c})}{\partial \mathbf{c}},
\end{aligned}
$$

where we have noted from (1.2) and (1.10) that $\mathbf{j}=\underline{\mathbf{0}}$, together with the boundary and initial conditions (1.3) and (1.4).

We let $\mathbf{L}^{2}(\Omega)=\left[L^{2}(\Omega)\right]^{N}, \mathbf{H}^{1}(\Omega)=\left[H^{1}(\Omega)\right]^{N}$, and similarly $\underline{\mathbf{L}}^{2}(\Omega)=\left[L^{2}(\Omega)\right]^{d}, \underline{\mathbf{H}}^{j}(\Omega)=$ $\left[H^{j}(\Omega)\right]^{d}, j=1,2$. Moreover, we let $(\cdot, \cdot)$ denote the $L^{2}$-inner product over $\Omega$ with the natural extensions to vector and matrix valued functions, e.g., for $N \times M$ matrices $A, B$ with entries in $L^{2}(\Omega)$ we let $(A, B)=\sum_{i=1}^{N} \sum_{j=1}^{M}\left(A_{i j}, B_{i j}\right)$. The $L^{2}$-norm will be denoted as $\|\cdot\|$, the $H^{1}$ norm as $\|\cdot\|_{1}$, the $L^{\infty}$-norm as $\|\cdot\|_{\infty}$. The same notations will be used for the corresponding norms of vector valued functions. In addition, the duality product between $\left(H^{1}(\Omega)\right)^{\prime}$ and $H^{1}(\Omega)$ will be denoted by $\langle\cdot, \cdot\rangle$, and similarly for vector valued functions. 
We introduce the following function spaces

$$
\begin{aligned}
\underline{\mathcal{V}} & =\left\{\underline{\mathbf{v}} \in \underline{\mathbf{C}}_{0}^{\infty}(\Omega) ; \quad \nabla \cdot \underline{\mathbf{v}}=0\right\}, \\
\underline{\mathbf{V}} & =\left\{\underline{\mathbf{v}} \in \underline{\mathbf{H}}_{0}^{1}(\Omega) ; \quad \nabla \cdot \underline{\mathbf{v}}=0\right\}, \\
\underline{\mathbf{H}} & =\left\{\underline{\mathbf{v}} \in \underline{\mathbf{L}}^{2}(\Omega) ; \quad \nabla \cdot \underline{\mathbf{v}}=0 \text { weakly in } \Omega,\left.\quad \underline{\mathbf{v}} \cdot \underline{\mathbf{n}}\right|_{\partial \Omega}=0\right\}, \\
\mathbf{H}_{+}^{1} & =\left\{\boldsymbol{\phi} \in\left[H^{1}(\Omega)\right]^{N} ; \quad \boldsymbol{\phi} \geq \mathbf{0}\right\}, \\
\mathbf{K} & =\left\{\boldsymbol{\phi} \in \mathbf{H}_{+}^{1} ; \quad \mathbf{1}^{T} \boldsymbol{\phi}=1\right\} .
\end{aligned}
$$

Note that $\underline{\mathbf{V}}$ and $\underline{\mathbf{H}}$ are obtained as the closures of $\underline{\mathcal{V}}$ in the $H^{1}$ and $L^{2}$ norms, respectively.

Definition 1 (Weak solution). Let $T>0$ and suppose that $\underline{\mathbf{u}}^{0} \in \underline{\mathbf{H}}, \mathbf{c}^{0} \in \mathbf{K}$ and $\underline{\mathbf{g}} \in$ $L^{2}\left(0, T ; \underline{\mathbf{L}}^{2}(\Omega)\right)$. A weak solution of (3.1) with (1.3) and (1.4) is given by functions

$$
\begin{aligned}
\underline{\mathbf{u}} & \in L^{2}(0, T ; \underline{\mathbf{V}}) \cap L^{\infty}(0, T ; \underline{\mathbf{H}}), \\
\mathbf{c} & \in L^{\infty}(0, T ; \mathbf{K}) \cap H^{1}\left(0, T,\left(\mathbf{H}^{1}(\Omega)\right)^{\prime}\right), \\
\mathbf{w} & \in L^{2}\left(0, T ; \mathbf{H}^{1}(\Omega)\right),
\end{aligned}
$$

such that

$$
\begin{gathered}
-\int_{0}^{T}\left(\underline{\mathbf{u}}, \partial_{t} \underline{\mathbf{v}}\right)+\int_{0}^{T}(\underline{\mathbf{u}} \cdot \nabla \underline{\mathbf{u}}, \underline{\mathbf{v}})+\int_{0}^{T}(2 \mu(\mathbf{c}) D(\underline{\mathbf{u}}), D(\underline{\mathbf{v}})) \\
=\left(\underline{\mathbf{u}}^{0}, \underline{\mathbf{v}}(0)\right)+\int_{0}^{T}(\underline{\mathbf{g}}, \underline{\mathbf{v}})-\lambda \int_{0}^{T}\left((\nabla \mathbf{w})^{T} \mathbf{c}, \underline{\mathbf{v}}\right), \\
\int_{0}^{T}\left\langle\partial_{t} \mathbf{c}, \boldsymbol{\psi}\right\rangle+\int_{0}^{T}(M \nabla \mathbf{w}, \nabla \boldsymbol{\psi})=\int_{0}^{T}(\mathbf{c} \otimes \underline{\mathbf{u}}, \nabla \boldsymbol{\psi}), \\
\int_{0}^{T}(\mathbf{w}, \boldsymbol{\phi}-\mathbf{c}) \leq \varepsilon \int_{0}^{T}(\nabla \mathbf{c}, \nabla(\boldsymbol{\phi}-\mathbf{c}))-\varepsilon^{-1} \int_{0}^{T}(A \mathbf{c}, \boldsymbol{\phi}-\mathbf{c}),
\end{gathered}
$$

for all $(\underline{\mathbf{v}}, \boldsymbol{\psi}, \boldsymbol{\phi}) \in H^{1}(0, T ; \underline{\mathbf{V}}) \times L^{2}\left(0, T ; \mathbf{H}^{1}(\Omega)\right) \times L^{2}(0, T ; \mathbf{K})$ with $\underline{\mathbf{v}}(T)=0$.

3.1. Numerical approximation. Let $\Omega \subset \mathbb{R}^{d}$ be an open bounded polyhedral domain and let $\left\{\mathcal{T}_{h}\right\}_{h>0}$ be a quasi-uniform partitioning of $\Omega$ into disjoint open simplices $\sigma$ with $h_{\sigma}=$ $\operatorname{diam}(\sigma)$ and $h=\max _{\sigma \in \mathcal{T}_{h}} h_{\sigma}$, so that $\bar{\Omega}=\cup_{\sigma \in \mathcal{T}_{h}} \bar{\sigma}$. Let $\mathcal{P}^{m}(\sigma)$ denote the space of polynomials of degree $\leq m$ on $\sigma$. Associated with $\mathcal{T}_{h}$ are the finite element spaces

$$
\begin{aligned}
S_{h} & =\left\{\phi \in C(\bar{\Omega}) ;\left.\phi\right|_{\sigma} \in \mathcal{P}^{1}(\sigma) \forall \sigma \in \mathcal{T}_{h}\right\}, \\
\mathbf{S}_{h} & =\left[S_{h}\right]^{N}, \\
\mathbf{S}_{h}^{+} & =\left\{\boldsymbol{\phi} \in \mathbf{S}_{h} ; \phi_{i} \geq 0 \text { for } i=1, \ldots, N\right\}, \\
\mathbf{K}_{h} & =\left\{\boldsymbol{\phi} \in \mathbf{S}_{h} ; \mathbf{1}^{T} \boldsymbol{\phi}=1\right\}, \\
\mathbf{K}_{h}^{0} & =\left\{\boldsymbol{\phi} \in \mathbf{K}_{h} ; \int_{\Omega} \boldsymbol{\phi}=\int_{\Omega} \mathbf{c}^{0}\right\}, \\
\underline{\mathbf{W}}_{h} & =\left\{\underline{\mathbf{v}} \in[C(\bar{\Omega})]^{d} \cap \underline{\mathbf{H}}_{0}^{1}(\Omega) ;\left.\underline{\mathbf{v}}\right|_{\sigma} \in \mathcal{P}^{2}(\sigma) \forall \sigma \in \mathcal{T}_{h}\right\}, \\
\underline{\mathbf{V}}_{h} & =\left\{\underline{\mathbf{v}} \in \underline{\mathbf{W}}_{h} ;(\nabla \cdot \underline{\mathbf{v}}, q)=0, \forall q \in S_{h}\right\} .
\end{aligned}
$$

Note that the pair $\underline{\mathbf{W}}_{h} \times S_{h}$ is the classical P2-P1 lowest order Taylor-Hood element for the discretization of Navier-Stokes equations, which satisfies the inf-sup condition. 
We define the orthogonal $\underline{\mathbf{L}}^{2}$ and $\underline{\mathbf{H}}^{1}$-projections on to $\underline{\mathbf{V}}_{h}$, respectively, as

$$
\left(\underline{\mathbf{v}}-\mathbf{P}_{0}^{h} \underline{\mathbf{v}}, \underline{\mathbf{w}}\right)=0 \quad \forall \underline{\mathbf{w}} \in \underline{\mathbf{V}}_{h} \quad \text { and } \quad\left(\nabla\left[\underline{\mathbf{v}}-\mathbf{P}_{1}^{h} \underline{\mathbf{v}}\right], \nabla \underline{\mathbf{w}}\right)=0 \quad \forall \underline{\mathbf{w}} \in \underline{\mathbf{V}}_{h} .
$$

We note that following error estimates, see for instance $[2,18]$

$$
\begin{array}{r}
\left\|\underline{\mathbf{v}}-\mathbf{P}_{1}^{h} \underline{\mathbf{v}}\right\|+h\left\|\nabla\left[\underline{\mathbf{v}}-\mathbf{P}_{1}^{h} \underline{\mathbf{v}}\right]\right\| \leq C h^{j}\|\underline{\mathbf{v}}\|_{\underline{\mathbf{H}}^{j}}, \\
\left\|\underline{\mathbf{v}}-\mathbf{P}_{0 \underline{\mathbf{v}}}^{h}\right\| \leq C h^{j}\|\underline{\mathbf{v}}\|_{\underline{\mathbf{H}}^{j}},
\end{array}
$$

for $\underline{\mathbf{v}} \in \underline{\mathbf{V}} \cap \underline{\mathbf{H}}^{j}(\Omega), j=1,2$. Furthermore, $\mathbf{P}_{0}^{h}$ is $\underline{\mathbf{H}}^{1}$ stable on $\underline{\mathbf{V}}$, see [18].

We also introduce the nodal interpolation operator $I^{h}: C(\bar{\Omega}) \rightarrow S_{h}$ and note the standard interpolation estimate

$$
\left\|\phi-I^{h} \phi\right\|+h\left\|\nabla\left[\phi-I^{h} \phi\right]\right\| \leq C h^{2}\|\phi\|_{H^{2}(\Omega)} .
$$

We naturally extend the definition of $I^{h}$ to vector valued functions. We also define the discrete inner product $(\phi, \psi)_{h}=\int_{\Omega} I^{h}(\phi \psi)$. We recall the well-known estimate

$$
\left|(\phi, \psi)-(\phi, \psi)_{h}\right| \leq C h\|\phi\|\|\psi\|_{1} \quad \forall \phi, \psi \in S_{h} .
$$

With the discrete inner product we associate the norm $\|\phi\|_{h}=\left[(\phi, \phi)_{h}\right]^{\frac{1}{2}}$ and recall its equivalence with the $L^{2}$ norm for all $\phi_{h} \in S_{h}$

$$
\left\|\phi_{h}\right\|^{2} \leq\left\|\phi_{h}\right\|_{h}^{2} \leq(d+2)\left\|\phi_{h}\right\|^{2} .
$$

The discrete $L^{2}$-projection $\mathbf{Q}^{h}: \mathbf{L}^{2}(\Omega) \rightarrow \mathbf{S}^{h}$ is defined as

$$
\left(\mathbf{Q}^{h} \mathbf{v}, \mathbf{w}\right)_{h}=(\mathbf{v}, \mathbf{w}) \quad \forall \mathbf{w} \in \mathbf{S}^{h},
$$

and satisfies the following estimate, cf. [7, Eq. (2.20)]

$$
\left\|\mathbf{v}-\mathbf{Q}^{h} \mathbf{v}\right\|+h\left\|\nabla\left[\mathbf{v}-\mathbf{Q}^{h} \mathbf{v}\right]\right\| \leq C h\|\nabla \mathbf{v}\| \quad \forall \mathbf{v} \in \mathbf{H}^{1}(\Omega) .
$$

Let

$$
\begin{aligned}
\mathcal{F}_{h} & =\left\{\boldsymbol{\psi} \in \mathbf{L}^{2}(\Omega) ; \int_{\Omega} \boldsymbol{\psi}=\mathbf{0},\left(\mathbf{1}^{T} \boldsymbol{\psi}, w\right)=0, \forall w \in S_{h}\right\}, \\
\widehat{\mathcal{F}}_{h} & =\left\{\boldsymbol{\psi} \in \mathbf{C}(\bar{\Omega}) ; \int_{\Omega} \boldsymbol{I}^{h} \boldsymbol{\psi}=\mathbf{0}, \mathbf{1}^{T} \boldsymbol{\psi}\left(\underline{\mathbf{x}}_{\ell}\right)=0 \text { for } \ell=1, \ldots, L\right\}, \\
\mathcal{V}_{h} & =\widehat{\mathcal{F}}_{h} \cap \mathbf{S}_{h},
\end{aligned}
$$

where $\left\{\underline{\mathbf{x}}_{\ell}\right\}_{\ell=1}^{L}$ is the set of all the nodes of $\mathcal{T}_{h}$. Then the discrete Green's operators $\mathcal{G}_{M}^{h}$ : $\mathcal{F}_{h} \rightarrow \mathcal{V}_{h}, \widehat{\mathcal{G}}_{M}^{h}: \widehat{\mathcal{F}}_{h} \rightarrow \mathcal{V}_{h}$ are defined as

$$
\begin{aligned}
& \left(M \nabla\left[\mathcal{G}_{M}^{h} \mathbf{v}\right], \nabla \mathbf{w}\right)=(\mathbf{v}, \mathbf{w}) \quad \forall \mathbf{w} \in \mathbf{S}_{h}, \\
& \left(M \nabla\left[\widehat{\mathcal{G}}_{M}^{h} \mathbf{v}\right], \nabla \mathbf{w}\right)=(\mathbf{v}, \mathbf{w})_{h} \quad \forall \mathbf{w} \in \mathbf{S}_{h} .
\end{aligned}
$$

We note that there exist constants $\lambda_{\max }^{M}, \lambda_{\text {min }}^{M}>0$, such that (cf. [7, Eqns. (2.7), (2.9)])

$$
\lambda_{\text {min }}^{M}\|\boldsymbol{\xi}\|_{\ell^{2}} \leq \boldsymbol{\xi} M \boldsymbol{\xi}^{T} \leq \lambda_{\text {max }}^{M}\|\boldsymbol{\xi}\|_{\ell^{2}},
$$

holds for all $\boldsymbol{\xi} \in \mathbb{R}^{N}$ with $\mathbf{1}^{T} \boldsymbol{\xi}=0$ with the mobility defined by (1.11) as well as (1.12). Hence, the well-posedness of $\mathcal{G}_{M}^{h}$ and $\widehat{\mathcal{G}}_{M}^{h}$ follows in both cases as in [3, 7].

For our numerical approximation we consider the splitting

$$
A \equiv A^{+}+A^{-}, \quad \text { where } A^{+(-)} \text {is symmetric positive (negative) semi-definite. }
$$


We construct an equidistant partition $\left\{t_{k}\right\}_{k=0}^{K}, t_{k}=k \tau$ of the time interval $[0, T]$ into subintervals $\left[t_{k-1}, t_{k}\right], k=1, \ldots, K$ with a step size $\tau=T / K$ and denote $d_{t} U^{k}=\frac{U^{k}-U^{k-1}}{\tau}$. We set $\mathbf{C}^{0}=\mathbf{Q}^{h} \mathbf{c}^{0} \in \mathbf{K}_{h}^{0}$ and $\underline{\mathbf{U}}^{0}=\mathbf{P}_{0}^{h} \underline{\mathbf{u}}^{0} \in \underline{\mathbf{V}}_{h}$.

For $k=1, \ldots, K$ we propose the following fully discrete numerical approximation of (3.1): Find $\left(\underline{\mathbf{U}}^{k}, P^{k}, \mathbf{W}^{k}, \mathbf{C}^{k}\right) \in \underline{\mathbf{W}}_{h} \times S_{h} \times \mathbf{S}_{h} \times \mathbf{S}_{h}^{+}$such that

$$
\left(d_{t} \underline{\mathbf{U}}^{k}, \underline{\mathbf{v}}\right)+\frac{1}{2}\left\{\left(\left[\underline{\mathbf{U}}^{k-1} \cdot \nabla\right] \underline{\mathbf{U}}^{k}, \underline{\mathbf{v}}\right)-\left(\left[\underline{\mathbf{U}}^{k-1} \cdot \nabla\right] \underline{\mathbf{v}}, \underline{\mathbf{U}}^{k}\right)\right\}
$$

$$
\begin{aligned}
+\left(2 \mu\left(\mathbf{C}^{k-1}\right) D\left(\underline{\mathbf{U}}^{k}\right), D(\underline{\mathbf{v}})\right)+\left(P^{k}, \nabla \cdot \underline{\mathbf{v}}\right) & =(\underline{\mathbf{g}}, \underline{\mathbf{v}})-\lambda\left(\left(\nabla \mathbf{W}^{k}\right)^{T} \mathbf{C}^{k-1}, \underline{\mathbf{v}}\right), \\
\left(\nabla \cdot \underline{\mathbf{U}}^{k}, q\right) & =0 \\
\left(d_{t} \mathbf{C}^{k}, \boldsymbol{\psi}\right)_{h}+\left(M \nabla \mathbf{W}^{k}, \nabla \boldsymbol{\psi}\right) & =\left(\mathbf{C}^{k-1} \otimes \underline{\mathbf{U}}^{k}, \nabla \boldsymbol{\psi}\right)
\end{aligned}
$$$$
\text { for all }(\underline{\mathbf{v}}, q, \boldsymbol{\psi}, \boldsymbol{\phi}) \in \underline{\mathbf{W}}_{h} \times S_{h} \times \mathbf{S}_{h} \times \mathbf{S}_{h}^{+} \text {. }
$$

In the next lemma we prove that the numerical solution exactly preserves the physically motivated constraints (2.8), (2.9).

Lemma 1. Let $\mathbf{C}^{0} \in \mathbf{K}_{h}^{0}$. Then the numerical solutions $\mathbf{C}^{k}, k=1, \ldots, K$, obtained by the scheme (3.10a-d) satisfy $\mathbf{C}^{k} \in \mathbf{K}_{h}^{0}$, i.e. in particular

$$
\text { (a) } \quad \int_{\Omega} \mathbf{C}^{k}=\int_{\Omega} \mathbf{c}^{0} \quad \text { and } \quad(b) \quad \mathbf{1}^{T} \mathbf{C}^{k}=1 .
$$

Proof. By definition $\mathbf{C}^{k} \in \mathbf{S}_{h}^{+}$, and so in order to show $\mathbf{C}^{k} \in \mathbf{K}_{h}^{0}$, we only need to prove (3.11). To show (3.11a) we set $\boldsymbol{\psi}=\mathbf{e}^{i}$ for $i=1, \ldots, N$ in (3.10c) and get

$$
\int_{\Omega} C_{i}^{k}=\left(\mathbf{C}^{k}, \mathbf{e}^{i}\right)_{h}=\left(\mathbf{C}^{k-1}, \mathbf{e}^{i}\right)_{h}=\int_{\Omega} C_{i}^{k-1}=\ldots=\int_{\Omega} C_{i}^{0}=\int_{\Omega} c_{i}^{0} .
$$

In order to prove (3.11b), we set $\boldsymbol{\psi}=\psi \mathbf{1}$ in (3.10c), for $\psi \in S^{h}$. Then we obtain, on recalling (1.10) and (3.10b), that

$$
\begin{aligned}
\left(d_{t} \mathbf{C}^{k}, \psi \mathbf{1}\right)_{h} & =-\left(M \nabla \mathbf{W}^{k}, \mathbf{1} \otimes \nabla \psi\right)+\left(\mathbf{C}^{k-1},[\mathbf{1} \otimes(\nabla \psi)] \underline{\mathbf{U}}^{k}\right) \\
& =\left(\left(\mathbf{C}^{k-1}\right)^{T} \mathbf{1},(\nabla \psi)^{T} \underline{\mathbf{U}}^{k}\right)=\left(\underline{\mathbf{U}}^{k}, \nabla \psi\right)=-\left(\nabla \cdot \underline{\mathbf{U}}^{k}, \psi\right)=0 \quad \forall \psi \in S^{h} .
\end{aligned}
$$

It immediately follows from (3.12) that

$$
\left(\mathbf{1}^{T} \mathbf{C}^{k}, \psi\right)_{h}=\left(\mathbf{1}^{T} \mathbf{C}^{k-1}, \psi\right)_{h} \quad \forall \psi \in S^{h},
$$

and hence $(3.11 \mathrm{~b})$ follows by induction.

Remark 1. The above proof implies that in order to preserve (3.11b), the finite element space used for the phase-field components $C_{i}^{k}$ should be a subset of the finite element space used for the pressure $P^{k}$. I.e., a compatibility condition needs to hold for the pressure-density finite element spaces: if $P^{k} \in U_{h}$ for some finite element space $U_{h}$, and if $\mathbf{C}^{k} \in \mathbf{S}_{h}=$ $\left[S_{h}\right]^{N}$, then we require $S_{h} \subset U_{h}$ in order to guarantee (3.11b). In addition, the pressuredensity compatibility condition is required for the well-posedness of a fixed point iteration for (3.10), and to be able to prove the existence of solutions to (3.10), see Remark 3 and the proof of Lemma 3 below. We also refer to [27, 2], where the pressure-density condition is required to guarantee a maximum principle for the discrete densities. Hence, the combination 
L'UBOMÍR BAŇAS AND ROBERT NÜRNBERG

of the pressure-density compatibility condition for $P^{k}, \mathbf{C}^{k}$ and the usual inf-sup condition for $\underline{\mathbf{U}}^{k}, P^{k}$ defines a velocity-pressure-density compatibility condition, which guarantees wellposedness of finite element discretizations of multicomponent flows. Note that the pressuredensity compatibility condition is not required in the case of binary fluids $N=2$, where (3.11b) is enforced implicitly when using the equivalent reformulation in terms of $c=c_{1}-c_{2}$, recall $\S 2.3$.

The next lemma shows that solutions for the scheme (3.10a-d) satisfy a discrete counterpart of the energy inequality (2.3).

Lemma 2. Let $\left\{\underline{\mathbf{U}}^{k}, P^{k}, \mathbf{C}^{k}, \mathbf{W}^{k}\right\}_{k=1}^{K}$ be a solution of (3.10a-d). Then the energy estimate

$$
\begin{gathered}
\frac{\varepsilon \lambda}{2}\left\|\nabla \mathbf{C}^{k}\right\|^{2}+\frac{\lambda}{\varepsilon}\left(\Psi\left(\mathbf{C}^{k}\right), 1\right)_{h}+\frac{1}{2}\left\|\underline{\mathbf{U}}^{k}\right\|^{2}+\tau \sum_{j=1}^{k}\left[2\left\|\mu^{\frac{1}{2}}\left(\mathbf{C}^{j-1}\right) D\left(\underline{\mathbf{U}}^{j}\right)\right\|^{2}+\lambda\left\|M^{\frac{1}{2}} \nabla \mathbf{W}^{j}\right\|^{2}\right] \\
+\sum_{j=1}^{k}\left[\frac{\lambda}{2}\left\|\nabla\left(\mathbf{C}^{j}-\mathbf{C}^{j-1}\right)\right\|^{2}+\frac{1}{2}\left\|\underline{\mathbf{U}}^{j}-\underline{\mathbf{U}}^{j-1}\right\|^{2}\right] \\
\leq \frac{\varepsilon \lambda}{2}\left\|\nabla \mathbf{C}^{0}\right\|^{2}+\frac{\lambda}{\varepsilon}\left(\Psi\left(\mathbf{C}^{0}\right), 1\right)_{h}+\frac{1}{2}\left\|\underline{\mathbf{U}}^{0}\right\|^{2}+\tau \sum_{j=1}^{k}\left(\underline{\mathbf{g}}, \underline{\mathbf{U}}^{j}\right)
\end{gathered}
$$

holds for all $k=1, \ldots, K$.

Proof. To prove the energy estimate we set $\underline{\mathbf{v}}=\underline{\mathbf{U}}^{k}, q=P^{k}, \boldsymbol{\psi}=\mathbf{W}^{k}, \boldsymbol{\phi}=\mathbf{C}^{k-1}$ in (3.10a)-(3.10d) to obtain

$$
\begin{aligned}
& \left(d_{t} \underline{\mathbf{U}}^{k}, \underline{\mathbf{U}}^{k}\right)+2\left\|\mu^{\frac{1}{2}}\left(\mathbf{C}^{k-1}\right) D\left(\underline{\mathbf{U}}^{k}\right)\right\|^{2}+\left(P^{k}, \nabla \cdot \underline{\mathbf{U}}^{k}\right)=\left(\underline{\mathbf{g}}, \underline{\mathbf{U}}^{k}\right)-\lambda\left(\left(\nabla \mathbf{W}^{k}\right)^{T} \mathbf{C}^{k-1}, \underline{\mathbf{U}}^{k}\right), \\
& \left(\nabla \cdot \underline{\mathbf{U}}^{k}, P^{k}\right)=0, \\
& \left(M \nabla \mathbf{W}^{k}, \nabla \mathbf{W}^{k}\right)=-\left(d_{t} \mathbf{C}^{k}, \mathbf{W}^{k}\right)_{h}+\left(\mathbf{C}^{k-1} \otimes \underline{\mathbf{U}}^{k}, \nabla \mathbf{W}^{k}\right), \\
& \varepsilon\left(\nabla \mathbf{C}^{k}, \nabla d_{t} \mathbf{C}^{k}\right)-\varepsilon^{-1}\left[\left(A^{-} \mathbf{C}^{k}, d_{t} \mathbf{C}^{k}\right)_{h}+\left(A^{+} \mathbf{C}^{k-1}, d_{t} \mathbf{C}^{k}\right)_{h}\right] \leq\left(\mathbf{W}^{k}, d_{t} \mathbf{C}^{k}\right)_{h} .
\end{aligned}
$$

Next, we multiply the last two equations above by $\lambda$, sum all the equations and get

$$
\begin{aligned}
& \left(d_{t} \underline{\mathbf{U}}^{k}, \underline{\mathbf{U}}^{k}\right)+2\left\|\mu^{\frac{1}{2}}\left(\mathbf{C}^{k-1}\right) D\left(\underline{\mathbf{U}}^{k}\right)\right\|^{2}+\lambda\left\|M^{\frac{1}{2}} \nabla \mathbf{W}^{k}\right\|^{2} \\
& \quad+\lambda \varepsilon\left(\nabla \mathbf{C}^{k}, \nabla d_{t} \mathbf{C}^{k}\right)-\lambda \varepsilon^{-1}\left(A^{-} \mathbf{C}^{k}+A^{+} \mathbf{C}^{k-1}, d_{t} \mathbf{C}^{k}\right)_{h} \leq\left(\underline{\mathbf{g}}, \underline{\mathbf{U}}^{k}\right) .
\end{aligned}
$$

From (3.13) and Lemma 1 it follows that

$$
\begin{aligned}
\frac{1}{2} \| \underline{\mathbf{U}}^{k} & \left\|^{2}+\frac{1}{2}\right\| \underline{\mathbf{U}}^{k}-\underline{\mathbf{U}}^{k-1}\left\|^{2}-\frac{1}{2}\right\| \underline{\mathbf{U}}^{k-1}\left\|^{2}+2 \tau\right\| \mu^{\frac{1}{2}}\left(\mathbf{C}^{k-1}\right) D\left(\underline{\mathbf{U}}^{k}\right) \|^{2} \\
& +\lambda \tau\left\|M^{\frac{1}{2}} \nabla \mathbf{W}^{k}\right\|^{2}+\frac{\varepsilon \lambda}{2}\left\|\nabla \mathbf{C}^{k}\right\|^{2}+\frac{\varepsilon \lambda}{2} \| \nabla\left(\mathbf{C}^{k}-\mathbf{C}^{k-1}\left\|^{2}-\frac{\varepsilon \lambda}{2}\right\| \nabla \mathbf{C}^{k-1} \|^{2}\right. \\
& +\frac{\lambda}{\varepsilon}\left(\Psi\left(\mathbf{C}^{k}\right), 1\right)_{h}-\frac{\lambda}{\varepsilon}\left(\Psi\left(\mathbf{C}^{k-1}\right), 1\right)_{h}+\frac{1}{2}\left(\left(A^{+}-A^{-}\right)\left(\mathbf{C}^{k}-\mathbf{C}^{k-1}\right), \mathbf{C}^{k}-\mathbf{C}^{k-1}\right)_{h} \\
\leq & \left(\underline{\mathbf{g}}, \underline{\mathbf{U}}^{k}\right),
\end{aligned}
$$

where we have employed the identity $2(\mathbf{a}-\mathbf{b})^{T} B \mathbf{a}=\mathbf{a}^{T} B \mathbf{a}+(\mathbf{a}-\mathbf{b})^{T} B(\mathbf{a}-\mathbf{b})-\mathbf{b}^{T} B \mathbf{b}$ for symmetric $N \times N$ matrices $B \in \mathbb{R}^{N \times N}$. Summing (3.14) for $k$ replaced by $j=1, \ldots, k$ yields the desired energy estimate. 
Remark 2. We define the following discrete counterpart of (1.6)

$$
\mathcal{E}_{c h}^{h}\left(\mathbf{C}^{k}\right)=\frac{\varepsilon}{2}\left\|\nabla \mathbf{C}^{k}\right\|^{2}+\frac{1}{\varepsilon}\left(\Psi\left(\mathbf{C}^{k}\right), 1\right)_{h},
$$

and the discrete Navier-Stokes energy

$$
\mathcal{E}_{n s}^{h}\left(\underline{\mathbf{U}}^{k}\right)=\frac{1}{2}\left\|\underline{\mathbf{U}}^{k}\right\|^{2} .
$$

Using the above notation, the energy inequality from Lemma 2 can be rewritten as

$$
\begin{aligned}
& \lambda \mathcal{E}_{c h}^{h}\left(\mathbf{C}^{k}\right)+\mathcal{E}_{n s}^{h}\left(\underline{\mathbf{U}}^{k}\right)+\lambda \tau \sum_{j=1}^{k}\left\|M^{\frac{1}{2}} \nabla \mathbf{W}^{j}\right\|^{2}+2 \tau \sum_{j=1}^{k}\left\|\mu^{\frac{1}{2}}\left(\mathbf{C}^{j-1}\right) D\left(\underline{\mathbf{U}}^{j}\right)\right\|^{2} \\
& \quad+\sum_{j=1}^{k}\left[\frac{\lambda}{2}\left\|\nabla\left(\mathbf{C}^{j}-\mathbf{C}^{j-1}\right)\right\|^{2}+\frac{1}{2}\left\|\underline{\mathbf{U}}^{j}-\underline{\mathbf{U}}^{j-1}\right\|^{2}\right] \leq \tau \sum_{j=1}^{k}\left(\underline{\mathbf{g}}, \underline{\mathbf{U}}^{j}\right)+\lambda \mathcal{E}_{c h}^{h}\left(\mathbf{C}^{0}\right)+\mathcal{E}_{n s}^{h}\left(\underline{\mathbf{U}}^{0}\right) .
\end{aligned}
$$

The nonlinear system (3.10a-d) is solved using the following fixed-point algorithm.

\section{Algorithm 1}

1. Given $\underline{\mathbf{U}}^{k-1} \in \underline{\mathbf{V}}_{h}, \mathbf{C}^{k-1} \in \mathbf{K}_{h}^{0}$ set $\underline{\mathbf{U}}^{k, 0}=\underline{\mathbf{U}}^{k-1}, \mathbf{C}^{k, 0}=\mathbf{C}^{k-1}$ and choose $\delta_{\text {fix }}>0$.

2. For $l \geq 1$ compute $\left(\underline{\mathbf{U}}^{k, l}, P^{k, l}, \mathbf{W}^{k, l}, \mathbf{C}^{k, l}\right) \in \underline{\mathbf{W}}_{h} \times S_{h} \times \mathbf{S}_{h} \times \mathbf{S}_{h}^{+}$such that

$$
\begin{aligned}
\left(\frac{\underline{\mathbf{U}}^{k, l}-\underline{\mathbf{U}}^{k-1}}{\tau}, \underline{\mathbf{v}}\right)+\frac{1}{2}\left\{\left(\left[\underline{\mathbf{U}}^{k-1} \cdot \nabla\right] \underline{\mathbf{U}}^{k, l}, \underline{\mathbf{v}}\right)\right. & \left.-\left(\left[\underline{\mathbf{U}}^{k-1} \cdot \nabla\right] \underline{\mathbf{v}}, \underline{\mathbf{U}}^{k, l}\right)\right\} \\
+\left(2 \mu\left(\mathbf{C}^{k-1}\right) D\left(\underline{\mathbf{U}}^{k, l}\right), D(\underline{\mathbf{v}})\right)+\left(P^{k, l}, \nabla \cdot \underline{\mathbf{v}}\right) & =(\underline{\mathbf{g}}, \underline{\mathbf{v}})-\lambda\left(\left(\nabla \mathbf{W}^{k, l}\right)^{T} \mathbf{C}^{k-1}, \underline{\mathbf{v}}\right), \\
\left(\nabla \cdot \underline{\mathbf{U}}^{k, l}, q\right) & =0, \\
\left(\frac{\mathbf{C}^{k, l}-\mathbf{C}^{k-1}}{\tau}, \boldsymbol{\psi}\right)_{h}+\left(M \nabla \mathbf{W}^{k, l}, \nabla \boldsymbol{\psi}\right) & =\left(\mathbf{C}^{k-1} \otimes \underline{\mathbf{U}}^{k, l-1}, \nabla \boldsymbol{\psi}\right),
\end{aligned}
$$

$$
\varepsilon\left(\nabla \mathbf{C}^{k, l}, \nabla\left(\boldsymbol{\phi}-\mathbf{C}^{k, l}\right)\right)-\left(\varepsilon^{-1} A^{-} \mathbf{C}^{k, l}+\mathbf{W}^{k, l}, \boldsymbol{\phi}-\mathbf{C}^{k, l}\right)_{h} \geq \varepsilon^{-1}\left(A^{+} \mathbf{C}^{k-1}, \boldsymbol{\phi}-\mathbf{C}^{k, l}\right)_{h},
$$

for all $(\underline{\mathbf{v}}, q, \boldsymbol{\psi}, \boldsymbol{\phi}) \in \underline{\mathbf{W}}_{h} \times S_{h} \times \mathbf{S}_{h} \times \mathbf{S}_{h}^{+}$.

3. If $\left\|\mathbf{C}^{k, l}-\mathbf{C}^{k, l-1}\right\|_{\infty}+\left\|\underline{\mathbf{U}}^{k, l}-\underline{\mathbf{U}}^{k, l-1}\right\|_{\infty} \leq \delta_{f i x}$ set $\underline{\mathbf{U}}^{k}=\underline{\mathbf{U}}^{k, l}, \mathbf{C}^{k, l}=\mathbf{C}^{k, l}$ and terminate; else set $l \rightarrow l+1$ and proceed to step 2 .

Let us fix $k \geq 1$. Then, for each $l \geq 1$ the system (3.15a-d) decouples into the NavierStokes part $(3.15 \mathrm{a}, \mathrm{b})$ and Cahn-Hilliard part $(3.15 \mathrm{c}, \mathrm{d})$. In each iteration of the fixed-point Algorithm 1 we first compute the solution $\left(\mathbf{C}^{k, l}, \mathbf{W}^{k, l}\right)$ of $(3.15 \mathrm{c}, \mathrm{d})$, which is then used in finding the solution $\left(\underline{\mathbf{U}}^{k, l}, P^{k, l}\right)$ of $(3.15 \mathrm{a}, \mathrm{b})$.

Remark 3. The pressure-density compatibility condition from Remark 1 implies that $\nabla \cdot\left[\mathbf{C}^{k-1} \otimes \underline{\mathbf{U}}^{k, l-1}\right] \in \mathcal{F}_{h}$. To see this, note that it follows from $\mathbf{C}^{k-1} \in \mathbf{K}_{h}$ and $\underline{\mathbf{U}}^{k, l-1} \in \underline{\mathbf{V}}_{h}$ that

$$
\left(\mathbf{1}^{T} \nabla \cdot\left[\mathbf{C}^{k-1} \otimes \underline{\mathbf{U}}^{k, l-1}\right], w\right)=\left(\nabla \cdot \underline{\mathbf{U}}^{k, l-1}, w\right)=0 \quad \forall w \in S_{h} .
$$

Hence, by (3.8a) it holds that

$$
\left(M \nabla \mathcal{G}_{M}^{h}\left(\nabla \cdot\left[\mathbf{C}^{k-1} \otimes \underline{\mathbf{U}}^{k, l-1}\right]\right), \nabla \boldsymbol{\psi}\right)=\left(\nabla \cdot\left[\mathbf{C}^{k-1} \otimes \underline{\mathbf{U}}^{k, l-1}\right], \boldsymbol{\psi}\right)=-\left(\mathbf{C}^{k-1} \otimes \underline{\mathbf{U}}^{k, l-1}, \nabla \boldsymbol{\psi}\right)
$$


for all $\boldsymbol{\psi} \in \mathbf{S}_{h}$. Consequently, we may rewrite (3.15c) as

$$
\left(\frac{\mathbf{C}^{k, l}-\mathbf{C}^{k-1}}{\tau}, \boldsymbol{\psi}\right)_{h}+\left(M \nabla \mathbf{W}^{k, l}, \nabla \boldsymbol{\psi}\right)=-\left(M \nabla \mathcal{G}_{M}^{h}\left(\nabla \cdot\left[\mathbf{C}^{k-1} \otimes \underline{\mathbf{U}}^{k, l-1}\right]\right), \nabla \boldsymbol{\psi}\right) .
$$

It follows, cf. $[8,4,7]$, that

$$
\mathbf{W}^{k, l}=-\widehat{\mathcal{G}}_{M}^{h}\left(\frac{\mathbf{C}^{k, l}-\mathbf{C}^{k-1}}{\tau}\right)+\Xi^{k, l} \mathbf{1}+\boldsymbol{\Lambda}^{k, l}-\mathcal{G}_{M}^{h}\left(\nabla \cdot\left[\mathbf{C}^{k-1} \otimes \underline{\mathbf{U}}^{k, l-1}\right]\right),
$$

with $\Xi^{k, l} \in S_{h}$ and $\boldsymbol{\Lambda}^{k, l}=\left(\Lambda_{1}^{k, l}, \ldots, \Lambda_{N}^{k, l}\right)^{T} \in \mathbb{R}^{N}$, with $\mathbf{1}^{T} \boldsymbol{\Lambda}^{k, l}=0$, are the Lagrange multipliers associated with the constraints (3.11b) and (3.11a), respectively. Hence, (3.15c,d) is equivalent to

$$
\begin{aligned}
\varepsilon( & \left.\nabla \mathbf{C}^{k, l}, \nabla\left(\boldsymbol{\phi}-\mathbf{C}^{k, l}\right)\right) \\
& -\left(\varepsilon^{-1} A^{-} \mathbf{C}^{k, l}-\widehat{\mathcal{G}}_{M}^{h}\left(\frac{\mathbf{C}^{k, l}-\mathbf{C}^{k-1}}{\tau}\right)-\mathcal{G}_{M}^{h}\left(\nabla \cdot\left[\mathbf{C}^{k-1} \otimes \underline{\mathbf{U}}^{k, l-1}\right]\right), \boldsymbol{\phi}-\mathbf{C}^{k, l}\right)_{h} \\
\geq & \left(\Xi^{k, l} \mathbf{1}+\mathbf{\Lambda}^{k, l}+\varepsilon^{-1} A^{+} \mathbf{C}^{k-1}, \boldsymbol{\phi}-\mathbf{C}^{k, l}\right)_{h} \quad \forall \boldsymbol{\phi} \in \mathbf{S}_{h}^{+} .
\end{aligned}
$$

We can now show that the fixed point iteration in Algorithm 1 is well-defined, and converges to a solution of (3.10).

Lemma 3. Given $\left(\underline{\mathbf{U}}^{k-1}, \mathbf{C}^{k-1}\right) \in \underline{\mathbf{V}}_{h} \times \mathbf{K}_{h}^{0}$, for each $l \geq 1$ there exists a solution $\left(\underline{\mathbf{U}}^{k, l}, P^{k, l}, \mathbf{W}^{k, l}, \mathbf{C}^{k, l}\right) \in \underline{\mathbf{V}}_{h} \times S_{h} \times \mathbf{S}_{h} \times \mathbf{K}_{h}^{0}$ to (3.15a-d). Here $\left(\underline{\mathbf{U}}^{k, l}, \mathbf{C}^{k, l}\right)$ is unique, and $P^{k, l}$ is unique up to an additive constant. Moreover, the fixed-point iteration in Algorithm 1 is a contraction. Consequently, there exists a solution $\left(\underline{\mathbf{U}}^{k}, P^{k}, \mathbf{W}^{k}, \mathbf{C}^{k}\right) \in \underline{\mathbf{V}}_{h} \times S_{h} \times \mathbf{S}_{h} \times \mathbf{K}_{h}^{0}$, to $(3.10 a-d)$.

Proof. We divide the proof into two parts. To simplify the presentation we assume that $\lambda=1$, $\mu_{\text {min }}=1, m_{0}=\lambda_{m i n}^{M}=1$ and $\underline{\mathbf{g}}=\underline{\mathbf{0}}$. However, it is straightforward to extend the proofs for the general case.

Note, that in general $\mathbf{W}^{k, l}$ is not unique. We denote by

$$
\widetilde{\mathbf{W}}^{k, l}=-\widehat{\mathcal{G}}_{M}^{h}\left(\frac{\mathbf{C}^{k, l}-\mathbf{C}^{k-1}}{\tau}\right)-\mathcal{G}_{M}^{h}\left(\nabla \cdot\left[\mathbf{C}^{k-1} \otimes \underline{\mathbf{U}}^{k, l-1}\right]\right),
$$

the uniquely defined part of $\mathbf{W}^{k, l}$, cf. (3.16), (3.8). Next, we observe that

$$
\begin{aligned}
& \left(\left(\nabla \mathbf{W}^{k, l}\right)^{T} \mathbf{C}^{k-1}, \underline{\mathbf{v}}\right)=\left(\left[\nabla\left(\widetilde{\mathbf{W}}^{k, l}+\Xi^{k, l} \mathbf{1}+\boldsymbol{\Lambda}^{k, l}\right)\right]^{T} \mathbf{C}^{k-1}, \underline{\mathbf{v}}\right) \\
& \quad=\left(\left[\nabla \widetilde{\mathbf{W}}^{k, l}+\mathbf{1} \otimes \nabla \Xi^{k, l}\right]^{T} \mathbf{C}^{k-1}, \underline{\mathbf{v}}\right)=\left(\left(\nabla \widetilde{\mathbf{W}}^{k, l}\right)^{T} \mathbf{C}^{k-1}, \underline{\mathbf{v}}\right) \quad \forall \underline{\mathbf{v}} \in \underline{\mathbf{V}}_{h} .
\end{aligned}
$$

Here we used the identity

$$
\left(\left[\mathbf{1} \otimes \nabla \Xi^{k, l}\right]^{T} \mathbf{C}^{k-1}, \underline{\mathbf{v}}\right)=\left(\left(\mathbf{1}^{T} \mathbf{C}^{k-1}\right) \nabla \Xi^{k, l}, \underline{\mathbf{v}}\right)=\left(\nabla \Xi^{k, l}, \underline{\mathbf{v}}\right)=-\left(\Xi^{k, l}, \nabla \cdot \underline{\mathbf{v}}\right)=0,
$$

which holds due to $\mathbf{C}^{k-1} \in \mathbf{K}_{h}, \Xi^{k, l} \in S_{h}, \underline{\mathbf{v}} \in \underline{\mathbf{V}}_{h}$ and the velocity-pressure-density compatibility condition. Hence, (3.19) implies that for $\underline{\mathbf{v}} \in \underline{\mathbf{V}}_{h}$ equation (3.15a) is equivalent to

$$
\begin{array}{r}
\left(\frac{\underline{\mathbf{U}}^{k, l}-\underline{\mathbf{U}}^{k-1}}{\tau}, \underline{\mathbf{v}}\right)+\frac{1}{2}\left\{\left(\left[\underline{\mathbf{U}}^{k-1} \cdot \nabla\right] \underline{\mathbf{U}}^{k, l}, \underline{\mathbf{v}}\right)-\left(\left[\underline{\mathbf{U}}^{k-1} \cdot \nabla\right] \underline{\mathbf{v}}, \underline{\mathbf{U}}^{k, l}\right)\right\} \\
\quad+\left(2 \mu\left(\mathbf{C}^{k-1}\right) D\left(\underline{\mathbf{U}}^{k, l}\right), D(\underline{\mathbf{v}})\right)=(\underline{\mathbf{g}}, \underline{\mathbf{v}})-\lambda\left(\left(\nabla \widetilde{\mathbf{W}}^{k, l}\right)^{T} \mathbf{C}^{k-1}, \underline{\mathbf{v}}\right) .
\end{array}
$$


The equivalence between the two formulations turns out to be convenient for the arguments below.

a) Existence of solutions for $(k, l)>0$. First, we derive an energy bound for $l>0$. We fix $k>0$ and assume that $\left(\mathbf{C}^{k-1}, \underline{\mathbf{U}}^{k-1}, \mathbf{W}^{k-1}\right) \in \mathbf{K}_{h}^{0} \times \underline{\mathbf{V}}_{h} \times \mathbf{S}_{h}$ satisfy the energy bound

$$
\frac{\varepsilon}{2}\left\|\nabla \mathbf{C}^{k-1}\right\|^{2}+\frac{1}{\varepsilon}\left(\Psi\left(\mathbf{C}^{k-1}\right), 1\right)_{h}+\frac{1}{2}\left\|\underline{\mathbf{U}}^{k-1}\right\|^{2}+\tau 2\left\|D\left(\underline{\mathbf{U}}^{k-1}\right)\right\|^{2}+\tau\left\|\nabla \widetilde{\mathbf{W}}^{k-1}\right\|^{2} \leq \tilde{C}
$$

for some fixed $\tilde{C}>0$. Next, we choose $(\underline{\mathbf{v}}, q, \boldsymbol{\psi}, \boldsymbol{\phi})=\left(\underline{\mathbf{U}}^{k, l}, P^{k, l}, \mathbf{W}^{k, l}, \mathbf{C}^{k-1}\right)$ in (3.15a)(3.15d), employ (3.19) and obtain

$$
\begin{aligned}
& \tilde{\mathcal{E}}^{k, l}= \frac{\varepsilon}{2}\left\|\nabla \mathbf{C}^{k, l}\right\|^{2}+\frac{1}{\varepsilon}\left(\Psi\left(\mathbf{C}^{k, l}\right), 1\right)_{h}+\frac{1}{2}\left\|\underline{\mathbf{U}}^{k, l}\right\|^{2}+\tau 2\left\|D\left(\underline{\mathbf{U}}^{k, l}\right)\right\|^{2}+\tau\left\|\nabla \widetilde{\mathbf{W}}^{k, l}\right\|^{2} \\
& \leq \frac{\varepsilon}{2}\left\|\nabla \mathbf{C}^{k-1}\right\|^{2}+\frac{1}{\varepsilon}\left(\Psi\left(\mathbf{C}^{k-1}\right), 1\right)_{h}+\frac{1}{2}\left\|\underline{\mathbf{U}}^{k-1}\right\|^{2} \\
& \quad+\tau\left(\mathbf{C}^{k-1} \otimes \underline{\mathbf{U}}^{k, l-1}, \nabla \widetilde{\mathbf{W}}\right. \\
&
\end{aligned}
$$

Since $\mathbf{C}^{k-1} \in \mathbf{K}_{h}$ we estimate the last term on the right-hand side using Cauchy-Schwarz and Young's inequalities as follows

$$
\tau\left(\left(\nabla \widetilde{\mathbf{W}}^{k, l}\right)^{T} \mathbf{C}^{k-1}, \underline{\mathbf{U}}^{k, l}\right) \leq \tau\left\|\mathbf{C}^{k-1}\right\|_{\infty}\left\|\nabla \widetilde{\mathbf{W}}^{k, l}\right\|\left\|\underline{\mathbf{U}}^{k, l}\right\| \leq \frac{\tau}{4}\left\|\nabla \widetilde{\mathbf{W}}^{k, l}\right\|^{2}+\tau\left\|\underline{\mathbf{U}}^{k, l}\right\|^{2},
$$

and similarly we bound the last but one term as

$$
\tau\left(\mathbf{C}^{k-1} \otimes \underline{\mathbf{U}}^{k, l-1}, \nabla \widetilde{\mathbf{W}}^{k, l}\right) \leq \tau\left\|\mathbf{C}^{k-1}\right\|_{\infty}\left\|\nabla \widetilde{\mathbf{W}}^{k, l}\right\|\left\|\underline{\mathbf{U}}^{k, l-1}\right\| \leq \frac{\tau}{4}\left\|\nabla \widetilde{\mathbf{W}}^{k, l}\right\|^{2}+\tau\left\|\underline{\mathbf{U}}^{k, l-1}\right\|^{2} .
$$

Using the previous two bounds we obtain for $\tau \leq \frac{1}{4}$ that

$$
\tilde{\mathcal{E}}^{k, l} \leq 2 \tilde{C}+\tau 4 \tilde{\mathcal{E}}^{k, l-1} .
$$

Hence, we conclude recursively for $\tau \leq \frac{1}{8}$ that

$$
\frac{\varepsilon}{2}\left\|\nabla \mathbf{C}^{k, l}\right\|^{2}+\frac{1}{\varepsilon}\left(\Psi\left(\mathbf{C}^{k, l}\right), 1\right)_{h}+\frac{1}{2}\left\|\underline{\mathbf{U}}^{k, l}\right\|^{2}+\tau 2\left\|D\left(\underline{\mathbf{U}}^{k, l}\right)\right\|^{2}+\tau\left\|\nabla \widetilde{\mathbf{W}}^{k, l}\right\|^{2} \leq 4 \tilde{C}
$$

for any $l \geq 0$.

We note that (3.17) for $\phi \in \mathbf{K}_{h}^{0}$ is the Euler-Lagrange equation of the strictly convex minimization problem, cf.:

$$
\begin{array}{r}
\min _{\mathbf{z}_{h} \in \mathbf{K}_{h}^{0}}\left\{\frac{\varepsilon}{2}\left\|\nabla \mathbf{z}_{h}\right\|^{2}-\frac{1}{2 \varepsilon}\left(A^{-} \mathbf{z}_{h}, \mathbf{z}_{h}\right)_{h}+\frac{1}{2 \tau}\left\|M^{\frac{1}{2}} \nabla\left[\widehat{\mathcal{G}}_{M}^{h}\left(\mathbf{z}_{h}-\mathbf{C}^{k-1}\right)\right]\right\|^{2}\right. \\
\left.-\frac{1}{\varepsilon}\left(A^{+} \mathbf{C}^{k-1}, \mathbf{z}_{h}\right)_{h}+\left(\mathcal{G}_{M}^{h}\left(\nabla \cdot\left[\mathbf{C}^{k-1} \otimes \underline{\mathbf{U}}^{k, l-1}\right]\right), \mathbf{z}_{h}\right)\right\},
\end{array}
$$

and the existence of unique $\mathbf{C}^{k, l} \in \mathbf{K}_{h}^{0}$, as well as the existence of suitable Lagrange multipliers $\Xi^{k, l}$ and $\boldsymbol{\Lambda}^{k, l}$, follows from standard optimization theory, see e.g. [7, Proof of Theorem 2.1]. The existence of $\mathbf{W}^{k, l} \in \mathbf{S}_{h}$ then follows from (3.16), and Remark 3 implies that $\left(\mathbf{C}^{k, l}, \mathbf{W}^{k, l}\right)$ is a solution to $(3.15 \mathrm{c}),(3.15 \mathrm{~d})$.

On noting the uniqueness of $\mathbf{C}^{k, l}$ and $\widetilde{\mathbf{W}}^{k, l} \equiv \widetilde{\mathbf{W}}^{k, l}\left(\mathbf{C}^{k, l}, \underline{\mathbf{U}}^{k, l-1}\right)$ and (3.21) the existence of a unique $\underline{\mathbf{U}}^{k, l} \in \underline{\mathbf{V}}_{h}$ in (3.20) (and hence also (3.15a)) follows by the Lax-Milgram theorem. Since the Taylor-Hood element $\underline{\mathbf{W}}_{h} \times S_{h}$ satisfies the inf-sup condition, the existence of $P^{k, l} \in S_{h}$, which is unique up to a constant, such that $\left(\underline{\mathbf{U}}^{k, l}, P^{k, l}\right)$ solves $(3.15 \mathrm{a}),(3.15 \mathrm{~b})$ follows from standard theory on the numerical approximation of Navier-Stokes equations, see for instance [30]. 
The above considerations imply that we may write $\left(\underline{\mathbf{U}}^{k, l}, \mathbf{C}^{k, l}\right)=\mathbf{F}^{k}\left(\underline{\mathbf{U}}^{k, l-1}, \mathbf{C}^{k, l-1}\right)$, where $\mathbf{F}^{k}: \underline{\mathbf{V}}_{h} \times \mathbf{K}_{h}^{0} \rightarrow \underline{\mathbf{V}}_{h} \times \mathbf{K}_{h}^{0}$ is a well-defined mapping represented implicitly by Algorithm 1 . Below we show that $\mathbf{F}^{k}$ is a contraction mapping and hence the Banach fixed point theorem yields the existence of the solution $\left(\underline{\mathbf{U}}^{k}, \mathbf{C}^{k}\right)$ as the unique fixed-point of the mapping $\mathbf{F}^{k}$.

b) Contraction property. We denote by $\mathbf{E}_{\boldsymbol{\phi}}^{k, l}=\phi^{k, l}-\phi^{k, l-1}$, and similarly for $\underline{E}_{\underline{\phi}}^{k, l}$ and $E_{\phi}^{k, l}$. Then subtracting (3.15a)-(3.15c), with $l$ replaced by $l-1$, from (3.15a)-(3.15c) yields

$$
\begin{aligned}
\left(\underline{E}_{\underline{U}}^{k, l}, \underline{\mathbf{v}}\right)+\frac{1}{2}\left\{\left(\left[\underline{\mathbf{U}}^{k-1} \cdot \nabla\right] \underline{E}_{\underline{U}}^{k, l}, \underline{\mathbf{v}}\right)-\left(\left[\underline{\mathbf{U}}^{k-1} \cdot \nabla\right] \underline{\mathbf{v}}, \underline{E}_{\underline{\mathbf{U}}}^{k, l}\right)\right\} & \\
+\left(2 \mu\left(\mathbf{C}^{k-1}\right) D\left(\underline{E}_{\underline{\mathbf{U}}}^{k, l}\right), D(\underline{\mathbf{v}})\right)+\left(E_{P}^{k, l}, \nabla \cdot \underline{\mathbf{v}}\right) & =-\left(\left(\nabla \mathbf{E}_{\widetilde{\mathbf{W}}}^{k, l}\right)^{T} \mathbf{C}^{k-1}, \underline{\mathbf{v}}\right), \\
\left(\nabla \cdot \underline{E}_{\underline{\mathbf{U}}}^{k, l}, q\right) & =0, \\
\left(\mathbf{E}_{\mathbf{C}}^{k, l}, \boldsymbol{\psi}\right)_{h}+\tau\left(M \nabla \mathbf{E}_{\widetilde{\mathbf{W}}}^{k, l}, \nabla \boldsymbol{\psi}\right) & =\tau\left(\mathbf{C}^{k-1} \otimes \underline{E}_{\underline{\mathbf{U}}}^{k, l-1}, \nabla \boldsymbol{\psi}\right) .
\end{aligned}
$$

Furthermore, setting $\phi=\mathbf{C}^{k, l-1}, \boldsymbol{\phi}=\mathbf{C}^{k, l}$ in (3.15d) for $l, l-1$, respectively, and addition of resulting inequalities gives

$$
\varepsilon\left\|\nabla \mathbf{E}_{\mathbf{C}}^{k, l}\right\|^{2}-\frac{1}{\varepsilon}\left(A^{-} \mathbf{E}_{\mathbf{C}}^{k, l}, \mathbf{E}_{\mathbf{C}}^{k, l}\right)_{h}-\left(\mathbf{E}_{\widetilde{\mathbf{W}}}^{k, l}, \mathbf{E}_{\mathbf{C}}^{k, l}\right)_{h} \leq 0 .
$$

On recalling that $A^{-}$is negative semi-definite and that $\boldsymbol{C}^{k, l}, \boldsymbol{C}^{k, l-1} \in \mathbf{K}_{h}^{0}$, we obtain from a Poincaré inequality that

$$
\varepsilon\left\|\mathbf{E}_{\mathbf{C}}^{k, l}\right\|^{2}-\left(\mathbf{E}_{\widetilde{\mathbf{W}}}^{k, l}, \mathbf{E}_{\mathbf{C}}^{k, l}\right)_{h} \leq 0 .
$$

Next, we set $\underline{\mathbf{v}}=\underline{E}_{\underline{\mathbf{U}}}^{k, l}, q=E_{P}^{k, l}$ in $(3.22 \mathrm{a}),(3.22 \mathrm{~b})$, respectively, integrate by parts in (3.22b) and add the resulting identities to obtain

$$
\frac{1}{\tau}\left\|\underline{E}_{\underline{\mathbf{U}}}^{k, l}\right\|^{2}+2\left\|D\left(\underline{E^{\mathbf{U}}}\right)\right\|^{2}=-\left(\left(\nabla \mathbf{E}_{\widetilde{\mathbf{W}}}^{k, l}\right)^{T} \mathbf{C}^{k-1}, \underline{E}_{\underline{\mathbf{U}}}^{k, l}\right) .
$$

We estimate the right-hand side of (3.24) using Cauchy-Schwarz and Young's inequalities as

$$
\begin{aligned}
\tau\left(\left(\nabla \mathbf{E}_{\widetilde{\mathbf{W}}}^{k, l}\right)^{T} \mathbf{C}^{k-1}, \underline{E}_{\underline{\mathbf{U}}}^{k, l}\right) & \leq \tau\left\|\nabla \mathbf{E}_{\widetilde{\mathbf{W}}}^{k, l}\right\|\left\|\mathbf{C}^{k-1}\right\|_{\infty}\left\|\underline{E^{k}} \underline{\underline{U}}\right\| \\
& \leq C \tau^{2}\left\|\nabla \mathbf{E}_{\widetilde{\mathbf{W}}}^{k, l}\right\|^{2}+\frac{1}{4}\left\|\underline{E_{\underline{\mathbf{U}}}^{k, l}}\right\| .
\end{aligned}
$$

After substituting (3.25) into (3.24) we obtain for sufficiently small $\tau$

$$
\left\|\underline{E}_{\underline{E}}^{k, l}\right\|^{2}+\tau 2\left\|D\left(\underline{E}_{\underline{\mathbf{U}}}^{k, l}\right)\right\|^{2} \leq C \tau^{2}\left\|\nabla \mathbf{E}_{\widetilde{\mathbf{W}}}^{k, l}\right\|^{2}+\frac{1}{4}\left\|\underline{E_{\underline{\mathbf{U}}}^{k, l}}\right\|^{2} \leq \frac{\tau}{4}\left\|\nabla \mathbf{E}_{\widetilde{\mathbf{W}}}^{k, l}\right\|^{2}+\frac{1}{4}\left\|\underline{E_{\underline{\mathbf{U}}}^{k, l}}\right\|^{2} .
$$

Next, we subtract $(3.15 \mathrm{c})$ for $l, l-1$ and set $\boldsymbol{\psi}=\mathbf{E}_{\widetilde{\mathbf{W}}}^{k, l}$

$$
\left(\mathbf{E}_{\mathbf{C}}^{k, l}, \mathbf{E}_{\widetilde{\mathbf{W}}}^{k, l}\right)_{h}+\tau\left(M \nabla \mathbf{E}_{\widetilde{\mathbf{W}}}^{k, l}, \nabla \mathbf{E}_{\widetilde{\mathbf{W}}}^{k, l}\right)=\tau\left(\mathbf{C}^{k-1} \otimes \underline{E}_{\underline{\mathbf{U}}}^{k, l-1}, \nabla \mathbf{E}_{\widetilde{\mathbf{W}}}^{k, l}\right) .
$$

We add together (3.27) and (3.23) and obtain

$$
\varepsilon\left\|\mathbf{E}_{\mathbf{C}}^{k, l}\right\|^{2}+\tau\left\|\nabla \mathbf{E}_{\widetilde{\mathbf{W}}}^{k, l}\right\|^{2} \leq \tau\left(\mathbf{C}^{k-1} \otimes \underline{E}_{\underline{\mathbf{U}}}^{k, l-1}, \nabla \mathbf{E}_{\widetilde{\mathbf{W}}}^{k, l}\right) .
$$

The right-hand side in the above expression is estimated using the fact that $\mathbf{C}^{k-1} \in \mathbf{K}^{h}$

$$
\begin{aligned}
\tau\left(\mathbf{C}^{k-1},\left(\nabla \mathbf{E}_{\widetilde{\mathbf{W}}}^{k, l}\right) \underline{E}_{\underline{\mathbf{U}}}^{k, l-1}\right) & \leq \tau\left\|\underline{E}_{\underline{\mathbf{U}}}^{k, l-1}\right\|\left\|\mathbf{C}^{k-1}\right\|_{\infty}\left\|\nabla \mathbf{E}_{\widetilde{\mathbf{W}}}^{k, l}\right\| \\
& \leq \frac{\tau}{4}\left\|\nabla \mathbf{E}_{\widetilde{\mathbf{W}}}^{k, l}\right\|^{2}+C \tau\left\|\underline{E}_{\underline{\mathbf{U}}}^{k, l-1}\right\|^{2} .
\end{aligned}
$$


Hence we obtain that

$$
\varepsilon\left\|\mathbf{E}_{\mathbf{C}}^{k, l}\right\|^{2}+\tau\left\|\nabla \mathbf{E}_{\widetilde{\mathbf{W}}}^{k, l}\right\|^{2} \leq \frac{\tau}{4}\left\|\nabla \mathbf{E}_{\widetilde{\mathbf{W}}}^{k, l}\right\|^{2}+C \tau\left\|\underline{E}_{\underline{\mathbf{U}}}^{k, l-1}\right\|^{2} .
$$

We add together (3.26) and (3.30) to obtain the inequality

$$
\left\|\underline{E}_{\underline{\mathbf{U}}}^{k, l}\right\|^{2}+\tau 2\left\|D\left(\underline{E}_{\underline{\mathbf{U}}}^{k, l}\right)\right\|^{2}+\varepsilon\left\|\mathbf{E}_{\mathbf{C}}^{k, l}\right\|^{2}+\tau\left\|\nabla \mathbf{E}_{\widetilde{\mathbf{W}}}^{k, l}\right\|^{2} \leq \frac{\tau}{2}\left\|\nabla \mathbf{E}_{\widetilde{\mathbf{W}}}^{k, l}\right\|^{2}+\frac{1}{4}\left\|\underline{E}_{\underline{\mathbf{U}}}^{k, l}\right\|^{2}+C \tau\left\|\underline{E}_{\underline{\mathbf{U}}}^{k, l-1}\right\|^{2} .
$$

The first two terms on the right hand side with index $l$ can be absorbed into the left hand side. Hence, we get

$$
\varepsilon\left\|\mathbf{E}_{\mathbf{C}}^{k, l}\right\|^{2}+\left\|\underline{E^{E}} \underline{\underline{U}}^{k, l}\right\|^{2}+\tau\left\|\nabla \mathbf{E}_{\widetilde{\mathbf{W}}}^{k, l}\right\|^{2} \leq C^{*} \tau\left\|\underline{E}_{\underline{\mathbf{U}}}^{k, l-1}\right\|^{2},
$$

and the fixed-point algorithm defines a contraction mapping for sufficiently small $\tau$. The Banach fixed point theorem then implies existence of unique limits and convergence $\underline{\mathbf{U}}^{k, l} \rightarrow \underline{\mathbf{U}}^{k}$ and $\mathbf{C}^{k, l} \rightarrow \mathbf{C}^{k}$ for $l \rightarrow \infty$. In addition, (3.31) implies the convergence $\widetilde{\mathbf{W}}^{k, l} \rightarrow \widetilde{\mathbf{W}}^{k}$, cf. (3.18). For the unique $\left(\underline{\mathbf{U}}^{k}, \mathbf{C}^{k}\right)$ the existence of $P^{k}$ and $\mathbf{W}^{k}=\widetilde{\mathbf{W}}^{k}+\Xi^{k} \mathbf{1}+\boldsymbol{\Lambda}^{k}$, such that $\left(\underline{\mathbf{U}}^{k}, P^{k}, \mathbf{W}^{k}, \mathbf{C}^{k}\right)$ solves (3.10), is implied by the inf-sup condition and Remark 3 , respectively.

Remark 4. Given a $\mathbf{W} \in \mathbf{S}_{h}$ we denote by $\sum \mathbf{W}=\frac{1}{N} \sum_{i=1}^{N} W_{i} \in S_{h}, f \mathbf{W}=|\Omega|^{-1} \int_{\Omega} \mathbf{W} \in$ $\mathbb{R}^{N}, \overline{\mathbf{W}}=\mathbf{W}-\mathbf{1} \sum \mathbf{W}, \widetilde{\mathbf{W}}=\overline{\mathbf{W}}-f \overline{\mathbf{W}}$ and note that $\mathbf{1}^{T} \widetilde{\mathbf{W}}=\mathbf{1}^{T} \overline{\mathbf{W}}=0, f \widetilde{\mathbf{W}}=0$. Due to the degeneracy of the mobility matrix $M$, it is not immediately obvious how to control the gradient of $\mathbf{W}^{k}$, since Lemma 2 and (3.9) only imply the bound $\left\|\nabla \overline{\mathbf{W}}^{k}\right\| \leq C$. However, cf. [4], it is possible to express

$$
\mathbf{W}^{k}=\widetilde{\mathbf{W}}^{k}+\Lambda^{k}+\mathbf{1} \Xi^{k}
$$

where $\boldsymbol{\Lambda}^{k} \in \mathbb{R}^{N}$ s.t. $\mathbf{1}^{T} \boldsymbol{\Lambda}^{k}=0$ and $\Xi^{k} \in S_{h}$ are Lagrange multipliers, recall also Remark 3 and the proof of Lemma 3. Since $\boldsymbol{\Lambda}^{k} \in \mathbb{R}^{N}$, we directly obtain a gradient bound for $\widetilde{\mathbf{W}}^{k}=\overline{\mathbf{W}}^{k}-\boldsymbol{\Lambda}^{k}$, i.e.

$$
\left\|\nabla \widetilde{\mathbf{W}}^{k}\right\| \leq C
$$

In addition, the estimate $|\Omega|^{-1}\left(\bar{W}_{i}^{k}, 1\right)=\Lambda_{i}^{k} \leq C, i=1, \ldots, N$, can be obtained similarly as in [4, Theorem 3.1]. Hence, a $\mathbf{H}^{1}(\Omega)$ bound for $\overline{\mathbf{W}}^{k}$ follows by the Poincaré inequality, i.e.

$$
\left\|\overline{\mathbf{W}}^{k}\right\|_{1} \leq C .
$$

Furthermore, we also note that for $\boldsymbol{\phi} \in \mathbf{K}_{h}$ it holds that $\left(\mathbf{1} \Xi^{k}, \boldsymbol{\phi}-\mathbf{C}^{k}\right)_{h}=0$. Hence for $\phi \in \mathbf{K}_{h}$ it follows from (3.10d) that

$$
\varepsilon\left(\nabla \mathbf{C}^{k}, \nabla\left(\boldsymbol{\phi}-\mathbf{C}^{k}\right)\right)-\left(\varepsilon^{-1} A^{-} \mathbf{C}^{k}+\overline{\mathbf{W}}^{k}, \boldsymbol{\phi}-\mathbf{C}^{k}\right)_{h} \geq \varepsilon^{-1}\left(A^{+} \mathbf{C}^{k-1}, \boldsymbol{\phi}-\mathbf{C}^{k}\right)_{h} .
$$

Lemma 4. The discrete time derivatives satisfy the following estimates

$$
\tau \sum_{j=1}^{K}\left\|d_{t} \underline{\mathbf{U}}^{j}\right\|_{\underline{\mathbf{V}}^{\prime}}^{\frac{4}{3}} \leq C \quad \text { and } \quad \tau \sum_{j=1}^{K}\left\|d_{t} \mathbf{C}^{j}\right\|_{\left(\mathbf{H}^{1}(\Omega)\right)^{\prime}}^{2} \leq C .
$$


Proof. Since $\left(d_{t} \underline{\mathbf{U}}^{k}, \mathbf{P}_{0}^{h} \underline{\mathbf{v}}\right)=\left(d_{t} \underline{\mathbf{U}}^{k}, \underline{\mathbf{v}}\right)$ we obtain $(\mathrm{cf}$. (3.20))

$$
\begin{aligned}
\left(d_{t} \underline{\mathbf{U}}^{k}, \underline{\mathbf{v}}\right)= & -\left(\left[\underline{\mathbf{U}}^{k-1} \cdot \nabla\right] \underline{\mathbf{U}}^{k}, \mathbf{P}_{0}^{h} \underline{\mathbf{v}}\right)+\left(\left[\underline{\mathbf{U}}^{k-1} \cdot \nabla\right] \mathbf{P}_{0}^{h} \underline{\mathbf{v}}, \underline{\mathbf{U}}^{k}\right)-\left(2 \mu\left(\mathbf{C}^{k-1}\right) D\left(\underline{\mathbf{U}}^{k}\right), D\left(\mathbf{P}_{0}^{h} \underline{\mathbf{v}}\right)\right) \\
& -\lambda\left(\left(\nabla \widetilde{\mathbf{W}}^{k}\right)^{T} \mathbf{C}^{k-1}, \mathbf{P}_{0}^{h} \underline{\mathbf{v}}\right) \\
\leq & \left\|\underline{\mathbf{U}}^{k-1}\right\|_{L^{3}}\left\|\nabla \underline{\mathbf{U}}^{k}\right\|\left\|\mathbf{P}_{0}^{h} \underline{\mathbf{v}}\right\|_{L^{6}}+\left\|\underline{\mathbf{U}}^{k-1}\right\|_{L^{4}}\left\|\underline{\mathbf{U}}^{k}\right\|_{L^{4}}\left\|\nabla \mathbf{P} \underline{\mathbf{P}}^{h}\right\| \\
& +C \mu_{\max }\left\|\nabla \underline{\mathbf{U}}^{k}\right\|\left\|\nabla \mathbf{P}{ }_{0}^{h} \underline{\mathbf{v}}\right\|+\lambda\left\|\mathbf{C}^{k-1}\right\|_{\infty}\left\|\nabla \widetilde{\mathbf{W}}^{k}\right\|\left\|\mathbf{P}_{0}^{h} \underline{\mathbf{v}}\right\| \\
\leq & C\left\|\nabla \mathbf{P}_{0}^{h} \underline{\mathbf{v}}\right\|\left\{\left\|\underline{\mathbf{U}}^{k-1}\right\| \frac{1}{2}\left\|\nabla \underline{\mathbf{U}}^{k-1}\right\|^{\frac{1}{2}}\left\|\nabla \underline{\mathbf{U}}^{k}\right\|\right. \\
& \left.+\left\|\underline{\mathbf{U}}^{k-1}\right\| \frac{1}{4}\left\|\nabla \underline{\mathbf{U}}^{k-1}\right\|^{\frac{3}{4}}\left\|\underline{\mathbf{U}}^{k}\right\|^{\frac{1}{4}}\left\|\nabla \underline{\mathbf{U}}^{k}\right\|^{\frac{3}{4}}+\left\|\nabla \underline{\mathbf{U}}^{k}\right\|+\left\|\nabla \widetilde{\mathbf{W}}^{k}\right\|\right\} \\
\leq & C\left\|\nabla \underline{\mathbf{v}}^{\|}\right\|\left\{\left\|\underline{\mathbf{U}}^{k-1}\right\|^{\frac{1}{2}}\left(\left\|\nabla \underline{\mathbf{U}}^{k-1}\right\|^{\frac{3}{2}}+\left\|\nabla \underline{\mathbf{U}}^{k}\right\|^{\frac{3}{2}}\right)\right. \\
& \left.+\left\|\underline{\mathbf{U}}^{k-1}\right\|^{\frac{1}{4}}\left\|\underline{\mathbf{U}}^{k}\right\|^{\frac{1}{4}}\left(\left\|\nabla \underline{\mathbf{U}}^{k-1}\right\|^{\frac{3}{2}}+\left\|\nabla \underline{\mathbf{U}}^{k}\right\|^{\frac{3}{2}}\right)+\left\|\nabla \underline{\mathbf{U}}^{k}\right\|+\left\|\nabla \widetilde{\mathbf{W}}^{k}\right\|\right\} .
\end{aligned}
$$

To estimate the nonlinear terms we employed the embedding $H^{1} \subset L^{6}$ and the interpolation inequality

$$
\|\underline{\mathbf{v}}\|_{L^{q}} \leq\|\underline{\mathbf{v}}\|_{L^{p}}^{1+d / q-d / p}\|\nabla \underline{\mathbf{v}}\|_{L^{p}}^{d / p-d / q},
$$

where $q \in[p, \infty)$ if $p=d$ and $q \in\left[p, \frac{d p}{d-p}\right]$ if $p<d$. The first estimate then follows from Lemma 2, Remark 4 and the stability of the projection $\mathbf{P}_{0}^{h}$. To obtain the second estimate we write

$$
\begin{aligned}
\left(d_{t} \mathbf{C}^{k}, \boldsymbol{\psi}\right)=\left(d_{t} \mathbf{C}^{k}, \mathbf{Q}^{h} \boldsymbol{\psi}\right)_{h} & =-\left(M \nabla \widetilde{\mathbf{W}} \widetilde{\mathbf{Q}}^{k}, \nabla \mathbf{Q}^{h} \boldsymbol{\psi}\right)+\left(\mathbf{C}^{k-1} \otimes \underline{\mathbf{U}}^{k}, \nabla \mathbf{Q}^{h} \boldsymbol{\psi}\right) \\
& \leq C\left\|\nabla \widetilde{\mathbf{W}}{ }^{k}\right\|\left\|\nabla \mathbf{Q}^{h} \boldsymbol{\psi}\right\|+\left\|\underline{\mathbf{U}}^{k}\right\|\left\|\mathbf{C}^{k-1}\right\|_{\infty}\left\|\nabla \mathbf{Q}^{h} \boldsymbol{\psi}\right\| .
\end{aligned}
$$

Hence, the second estimate follows from Lemma 2, Remark 4 and the stability of $\mathbf{Q}^{h}$ implied by $(3.7)$.

3.1.1. Convergence. Given the discrete solutions $\mathbf{C}^{k}, k=0, \ldots, K$, we define the piecewise constant interpolants as

$$
\begin{array}{ll}
\mathbf{C}^{+}(t)=\mathbf{C}^{k+1} & \text { for } t \in\left(t_{k}, t_{k+1}\right], \\
\mathbf{C}^{-}(t)=\mathbf{C}^{k} & \text { for } t \in\left(t_{k}, t_{k+1}\right],
\end{array}
$$

and the piecewise linear interpolant

$$
\mathbf{C}(t)=\frac{t-t_{k}}{\tau} \mathbf{C}^{k}+\frac{t_{k+1}-t}{\tau} \mathbf{C}^{k+1} \quad \text { for } \quad t \in\left[t_{k}, t_{k+1}\right] .
$$

Using analogous notations for the variables $\underline{\mathbf{U}}^{k}, P^{k}$ and $\mathbf{W}^{k}$, the fully discrete scheme (3.10a-d) can be restated as

$$
\begin{aligned}
&\left(\partial_{t} \underline{\mathbf{U}}, \underline{\mathbf{v}}\right)+ \frac{1}{2}\left\{\left(\left[\underline{\mathbf{U}}^{-} \cdot \nabla\right] \underline{\mathbf{U}}^{+}, \underline{\mathbf{v}}\right)+\left(\left[\underline{\mathbf{U}}^{-} \cdot \nabla\right] \underline{\mathbf{v}}, \underline{\mathbf{U}}^{+}\right)\right\} \\
&+2\left(\mu\left(\mathbf{C}^{-}\right) D\left(\underline{\mathbf{U}}^{+}\right), D(\underline{\mathbf{v}})\right)+\left(P^{+}, \nabla \cdot \underline{\mathbf{v}}\right)=(\underline{\mathbf{g}}, \underline{\mathbf{v}})-\lambda\left(\left(\nabla \mathbf{W}^{+}\right)^{T} \mathbf{C}^{-}, \underline{\mathbf{v}}\right), \\
&\left(\nabla \cdot \underline{\mathbf{U}}^{+}, q\right)=0, \\
&\left(\partial_{t} \mathbf{C}, \boldsymbol{\psi}\right)_{h}-\left(\mathbf{C}^{-} \otimes \underline{\mathbf{U}}^{+}, \nabla \boldsymbol{\psi}\right)+\left(M \nabla \mathbf{W}^{+}, \nabla \boldsymbol{\psi}\right)=0, \\
& \varepsilon\left(\nabla \mathbf{C}^{+}, \nabla\left(\boldsymbol{\phi}-\mathbf{C}^{+}\right)\right)-\left(\varepsilon^{-1} A^{-} \mathbf{C}^{+}+\mathbf{W}^{+}, \boldsymbol{\phi}-\mathbf{C}^{+}\right)_{h} \geq \varepsilon^{-1}\left(A^{+} \mathbf{C}^{-}, \boldsymbol{\phi}-\mathbf{C}^{+}\right)_{h} .
\end{aligned}
$$


Remark 5. By the estimates from Lemmas 1, 2 and 4 we obtain for all $h, \tau>0$ the boundedness of $\mathbf{C}^{ \pm}$in $L^{\infty}\left(0, T ; \mathbf{H}^{1}(\Omega)\right)$, $\partial_{t} \mathbf{C}$ in $L^{2}\left(0, T ;(\mathbf{H}(\Omega))^{\prime}\right), \underline{\mathbf{U}}^{ \pm}$in $L^{\infty}\left(0, T ; \underline{\mathbf{L}}^{2}(\Omega)\right) \cap$ $L^{2}\left(0, T ; \underline{\mathbf{H}}^{1}(\Omega)\right), \partial_{t} \underline{\mathbf{U}}$ in $L^{\frac{4}{3}}\left(0, T ; \underline{\mathbf{V}}^{\prime}\right)$. Furthermore, the boundedness of $\overline{\mathbf{W}}^{+}=\mathbf{W}^{+}-\mathbf{1} \boldsymbol{E} \mathbf{W}^{+}$ in $L^{2}\left(0, \bar{T} ; \mathbf{H}^{1}(\Omega)\right)$ follows from Remark 4 .

In addition, Lemma 2 implies that

$$
\left\|\mathbf{C}^{ \pm}-\mathbf{C}\right\|_{L^{2}\left(0, T ; \mathbf{L}^{2}(\Omega)\right)} \rightarrow 0
$$

and

$$
\left\|\underline{\mathbf{U}}^{ \pm}-\underline{\mathbf{U}}\right\|_{L^{2}\left(0, T ; \underline{\mathbf{L}}^{2}(\Omega)\right)} \rightarrow 0
$$

for $\tau \rightarrow 0$.

The next theorem shows the convergence of the numerical solutions to a weak solution.

Theorem 1. Let $\left(\underline{\mathbf{U}}^{0}, \mathbf{C}^{0}\right) \rightarrow\left(\underline{\mathbf{u}}^{0}, \mathbf{c}^{0}\right)$ in $\underline{\mathbf{L}}^{2}(\Omega) \times \mathbf{L}^{2}(\Omega)$ for $h \rightarrow 0$. Then there exists a subsequence $\{\underline{\mathbf{U}}, \mathbf{C}, \overline{\mathbf{W}}\}_{h, \tau \geq 0}$ of solutions of (3.33) which converges towards a limit $(\underline{\mathbf{u}}, \mathbf{c}, \mathbf{w}) \in$ $L^{2}(0, T ; \underline{\mathbf{V}}) \times L^{\infty}(0, T ; \mathbf{K}) \times L^{2}\left(0, T ; \mathbf{H}^{1}(\Omega)\right)$ for $h, \tau \rightarrow 0$. In addition, $(\underline{\mathbf{u}}, \mathbf{c}, \mathbf{w})$ is a weak solution of (1.1) in the sense of Definition 1.

Proof. By Remark 5 from the sequence $\{\underline{\mathbf{U}}, \mathbf{C}, \mathbf{W}\}_{h, \tau \geq 0}$ we can choose a subsequence such that

$$
\begin{array}{rlll}
\mathbf{C}, \mathbf{C}^{ \pm} \rightarrow \mathbf{c} & \text { weakly* } & \text { in } & L^{\infty}\left(0, T ; \mathbf{H}^{1}(\Omega)\right), \\
\partial_{t} \mathbf{C} \rightarrow \partial_{t} \mathbf{c} & \text { weakly } & \text { in } & L^{2}\left(0, T ;\left(\mathbf{H}^{1}(\Omega)\right)^{\prime}\right), \\
\overline{\mathbf{W}}^{+} \rightarrow \overline{\mathbf{w}} & \text { weakly } & \text { in } & L^{2}\left(0, T ; \mathbf{H}^{1}(\Omega)\right), \\
\underline{\mathbf{U}}, \underline{\mathbf{U}}^{ \pm} \rightarrow \underline{\mathbf{u}} & \text { weakly* } & \text { in } & L^{\infty}\left(0, T ; \underline{\mathbf{L}}^{2}(\Omega)\right), \\
\underline{\mathbf{U}}, \underline{\mathbf{U}}^{ \pm} \rightarrow \underline{\mathbf{u}} & \text { weakly } & \text { in } & L^{2}\left(0, T ; \underline{\mathbf{H}}^{1}(\Omega)\right), \\
\partial_{t} \underline{\mathbf{U}} \rightarrow \partial_{t} \underline{\mathbf{u}} & \text { weakly } & \text { in } & L^{\frac{4}{3}}\left(0, T ; \underline{\mathbf{V}}^{\prime}\right) .
\end{array}
$$

In addition, the estimates from Lemmas 2 and 4, together with an Aubin-Lions compactness argument, yield strong convergence

$$
\begin{array}{llll}
\mathbf{C}, \mathbf{C}^{ \pm} \rightarrow \mathbf{c} & \text { strongly } & \text { in } & L^{2}\left(0, T ; \mathbf{L}^{2}(\Omega)\right), \\
\underline{\mathbf{U}}, \underline{\mathbf{U}}^{ \pm} \rightarrow \underline{\mathbf{u}} & \text { strongly } & \text { in } & L^{2}\left(0, T ; \underline{\mathbf{L}}^{2}(\Omega)\right) .
\end{array}
$$

Furthermore, the strong convergence $(3.38)_{1}$ implies that $\mathbf{c} \in \mathbf{K}$. To show that the limit $(\underline{\mathbf{u}}, \mathbf{c}, \mathbf{w})$ satisfies $(3.2)$ we take $\left(\underline{\mathbf{v}}_{h}, q_{h}, \boldsymbol{\psi}_{h}, \boldsymbol{\phi}_{h}\right)=\left(\mathbf{P}_{1}^{h} \underline{\mathbf{v}}, I^{h} q, \boldsymbol{I}^{h} \boldsymbol{\psi}, \boldsymbol{I}^{h} \boldsymbol{\phi}\right)$ for $(\underline{\mathbf{v}}, q, \boldsymbol{\psi}, \boldsymbol{\phi}) \in$ $H^{1}(0, T ; \underline{\mathcal{V}}) \times C\left(0, T ; H^{2}(\Omega)\right) \times C\left(0, T ; \mathbf{H}^{2}(\Omega)\right) \times C\left(0, T ; \mathbf{H}_{+}^{1} \cap \mathbf{H}^{2}(\Omega)\right)$ in (3.10a)-(3.10d). By (3.4) and (3.3) we note that for $h \rightarrow 0$

$$
\left(\underline{\mathbf{v}}_{h}, q_{h}, \boldsymbol{\psi}_{h}, \boldsymbol{\phi}_{h}\right) \rightarrow(\underline{\mathbf{v}}, q, \boldsymbol{\psi}, \boldsymbol{\phi}),
$$

strongly in the corresponding $H^{1}$ norms for all $t \in(0, T)$.

First, we note that $(3.34),(3.37)_{5},(3.39)$ imply that

$$
0=\int_{0}^{T}(\nabla \cdot \underline{\mathbf{u}}, q)=\lim _{h, \tau \rightarrow 0} \int_{0}^{T}\left(\nabla \cdot \underline{\mathbf{U}}^{+}, q_{h}\right) .
$$

Hence, it follows that $\nabla \cdot \underline{\mathbf{u}}=0$, a.e. in $(0, T) \times \Omega$.

Since, $\underline{\mathbf{v}}_{h} \in \underline{\mathbf{V}}_{h}$ we note that for any $h$

$$
\int_{0}^{T}\left(P^{+}, \nabla \cdot \underline{\mathbf{v}}_{h}\right)=0,
$$


and hence the pressure term in (3.33) vanishes. Integration by parts in the time variable implies

$$
\int_{0}^{T}\left(\partial_{t} \underline{\mathbf{U}}, \underline{\mathbf{v}}_{h}\right)=-\int_{0}^{T}\left(\underline{\mathbf{U}}, \partial_{t} \underline{\mathbf{v}}_{h}\right)+\left(\underline{\mathbf{U}}(T), \underline{\mathbf{v}}_{h}(T)\right)-\left(\underline{\mathbf{U}}(0), \underline{\mathbf{v}}_{h}(0)\right),
$$

and by $(3.37)_{3},(3.39)$ we get with $\underline{\mathbf{v}}(T)=\underline{\mathbf{0}}$ that

$$
\int_{0}^{T}\left(\partial_{t} \underline{\mathbf{U}}, \underline{\mathbf{v}}_{h}\right) \rightarrow-\int_{0}^{T}\left(\underline{\mathbf{u}}, \partial_{t} \underline{\mathbf{v}}\right)-(\underline{\mathbf{u}}(0), \underline{\mathbf{v}}(0)) .
$$

Next, we show that

$$
\int_{0}^{T} \frac{1}{2}\left\{\left(\left[\underline{\mathbf{U}}^{-} \cdot \nabla\right] \underline{\mathbf{U}}^{+}, \underline{\mathbf{v}}_{h}\right)-\left(\left[\underline{\mathbf{U}}^{-} \cdot \nabla\right] \underline{\mathbf{v}}_{h}, \underline{\mathbf{U}}^{+}\right)\right\} \rightarrow \int_{0}^{T}([\underline{\mathbf{u}} \cdot \nabla] \underline{\mathbf{u}}, \underline{\mathbf{v}}) .
$$

We obtain after integration by parts in the second term that

$$
\begin{aligned}
& \frac{1}{2}\left\{\left(\left[\underline{\mathbf{U}}^{-} \cdot \nabla\right] \underline{\mathbf{U}}^{+}, \underline{\mathbf{v}}_{h}\right)-\left(\left[\underline{\mathbf{U}}^{-} \cdot \nabla\right] \underline{\mathbf{v}}_{h}, \underline{\mathbf{U}}^{+}\right)\right\} \\
& =\frac{1}{2}\left(\left[\underline{\mathbf{U}}^{-} \cdot \nabla\right] \underline{\mathbf{U}}^{+}, \underline{\mathbf{v}}_{h}\right)+\frac{1}{2}\left(\left[\underline{\mathbf{U}}^{-} \cdot \nabla\right] \underline{\mathbf{U}}^{+}, \underline{\mathbf{v}}_{h}\right)+\frac{1}{2}\left(\left[\nabla \cdot \underline{\mathbf{U}}^{-}\right] \underline{\mathbf{U}}^{+}, \underline{\mathbf{v}}_{h}\right) .
\end{aligned}
$$

Hence, we observe

$$
\begin{aligned}
& \int_{0}^{T}\left[\left(\left[\underline{\mathbf{U}}^{-} \cdot \nabla\right] \underline{\mathbf{U}}^{+}, \underline{\mathbf{v}}_{h}\right)-([\underline{\mathbf{u}} \cdot \nabla] \underline{\mathbf{u}}, \underline{\mathbf{v}})\right] \\
& \leq\left|\int_{0}^{T}\left(\left[\left(\underline{\mathbf{U}}^{-}-\underline{\mathbf{u}}\right) \cdot \nabla\right] \underline{\mathbf{U}}^{+}, \underline{\mathbf{v}}_{h}\right)\right|+\left|\int_{0}^{T}\left([\underline{\mathbf{u}} \cdot \nabla]\left(\underline{\mathbf{U}}^{+}-\underline{\mathbf{u}}\right), \underline{\mathbf{v}}_{h}\right)\right| \\
& +\left|\int_{0}^{T}\left([\underline{\mathbf{u}} \cdot \nabla] \underline{\mathbf{u}}, \underline{\mathbf{v}}_{h}-\underline{\mathbf{v}}\right)\right| \\
& \leq \int_{0}^{T}\left\|\left(\underline{\mathbf{U}}^{-}-\underline{\mathbf{u}}\right)\right\|\left\|\nabla \underline{\mathbf{U}}^{+}\right\|\left\|\underline{\mathbf{v}}_{h}\right\|_{\infty}+\left|\int_{0}^{T}\left([\underline{\mathbf{u}} \cdot \nabla]\left(\underline{\mathbf{U}}^{+}-\underline{\mathbf{u}}\right), \underline{\mathbf{v}}_{h}\right)\right| \\
& +\int_{0}^{T}\|\underline{\mathbf{u}}\|_{\underline{\mathbf{L}}^{4}}\|\nabla \underline{\mathbf{u}}\|\left\|\underline{\mathbf{v}}_{h}-\underline{\mathbf{v}}\right\|_{\underline{\mathbf{L}}^{4}} \\
& \leq \int_{0}^{T}\left\|\left(\underline{\mathbf{U}}^{-}-\underline{\mathbf{u}}\right)\right\|\left\|\nabla \underline{\mathbf{U}}^{+}\right\|\left\|\underline{\mathbf{v}}_{h}\right\|_{\infty}+\left|\int_{0}^{T}\left([\underline{\mathbf{u}} \cdot \nabla]\left(\underline{\mathbf{U}}^{+}-\underline{\mathbf{u}}\right), \underline{\mathbf{v}}_{h}\right)\right| \\
& +\max _{[0, T]}\left\|\underline{\mathbf{v}}_{h}-\underline{\mathbf{v}}\right\|_{1} \int_{0}^{T}\|\underline{\mathbf{u}}\|_{1}^{2} \\
& \rightarrow 0 \text { for } h, \tau \rightarrow 0 \text {, }
\end{aligned}
$$

since the first two term disappear by $(3.38)_{2},(3.37)_{4}$, respectively, and for the last term we use the continuous embedding $H^{1} \subset L^{4}$ and (3.39).

We obtain in a similar way that

$$
\frac{1}{2} \int_{0}^{T}\left(\left[\underline{\mathbf{U}}^{-} \cdot \nabla\right] \underline{\mathbf{U}}^{+}, \underline{\mathbf{v}}_{h}\right) \rightarrow \frac{1}{2} \int_{0}^{T}([\underline{\mathbf{u}} \cdot \nabla] \underline{\mathbf{u}}, \underline{\mathbf{v}}), \quad \text { and } \quad \frac{1}{2} \int_{0}^{T}\left(\left[\nabla \cdot \underline{\mathbf{U}}^{-}\right] \underline{\mathbf{U}}^{+}, \underline{\mathbf{v}}_{h}\right) \rightarrow 0,
$$

since $\nabla \cdot \underline{\mathbf{u}}=0$, a.e. in $(0, T) \times \Omega$. Hence, from the above results and (3.43) we conclude (3.42). Further, we obtain that

$$
\int_{0}^{T}\left(\left(\nabla \mathbf{W}^{+}\right)^{T} \mathbf{C}^{-}, \underline{\mathbf{v}}_{h}\right) \rightarrow \int_{0}^{T}\left((\nabla \mathbf{w})^{T} \mathbf{c}, \underline{\mathbf{v}}\right) \quad \text { for } \quad \tau, h \rightarrow 0,
$$



since by $(3.37)_{1,3},(3.38)_{1}$ and $\underline{\mathbf{v}}_{h} \rightarrow \underline{\mathbf{v}}$

$$
\begin{aligned}
\left|\int_{0}^{T}\left[\left(\left(\nabla \mathbf{W}^{+}\right)^{T} \mathbf{C}^{-}, \underline{\mathbf{v}}_{h}\right)-\left((\nabla \mathbf{w})^{T} \mathbf{c}, \underline{\mathbf{v}}_{h}\right)\right]\right| \leq & \left\|\nabla \mathbf{W}^{+}\right\|\left\|_{L^{2}\left(\mathbf{L}^{2}\right)}\right\| \mathbf{C}^{-}-\mathbf{c}\left\|_{L^{2}\left(\mathbf{L}^{2}\right)}\right\| \underline{\mathbf{v}}_{h} \|_{L^{\infty}\left(\Omega_{T}\right)} \\
& +\left|\int_{0}^{T}\left(\nabla \mathbf{W}^{+}-\nabla \mathbf{w}, \mathbf{c} \otimes \underline{\mathbf{v}}_{h}\right)\right| \rightarrow 0 .
\end{aligned}
$$

We observe that $(3.37)_{5}$ and $(3.38)_{1}$ imply

$$
\int_{0}^{T}\left(\mu\left(\mathbf{C}^{-}\right) D\left(\underline{\mathbf{U}}^{+}\right), D\left(\underline{\mathbf{v}}_{h}\right)\right) \rightarrow \int_{0}^{T}(\mu(\mathbf{c}) D(\underline{\mathbf{u}}), D(\underline{\mathbf{v}})) \quad \text { for } \quad h, \tau \rightarrow 0 .
$$

Collecting (3.40), (3.41), (3.42), (3.45), (3.44) together with the strong convergence $\underline{\mathbf{U}}^{0} \rightarrow \underline{\mathbf{u}}^{0}$ we verify that

$$
\begin{gathered}
-\int_{0}^{T}\left(\underline{\mathbf{u}}, \partial_{t} \underline{\mathbf{v}}\right)+\int_{0}^{T}(\underline{\mathbf{u}} \cdot \nabla \underline{\mathbf{u}}, \underline{\mathbf{v}})+\int_{0}^{T}(2 \mu(\mathbf{c}) D(\underline{\mathbf{u}}), D(\underline{\mathbf{v}})) \\
=\left(\underline{\mathbf{u}}^{0}, \underline{\mathbf{v}}(0)\right)+\int_{0}^{T}(\underline{\mathbf{g}}, \underline{\mathbf{v}})-\lambda \int_{0}^{T}\left((\nabla \mathbf{w})^{T} \mathbf{c}, \underline{\mathbf{v}}\right),
\end{gathered}
$$

for all $\underline{\mathbf{v}} \in H^{1}(0, T ; \underline{\mathcal{V}})$ with $\underline{\mathbf{v}}(T)=\underline{\mathbf{0}}$.

Similarly as in (3.44), we deduce the convergence

$$
\int_{0}^{T}\left(\mathbf{C}^{-} \otimes \underline{\mathbf{U}}^{+}, \nabla \boldsymbol{\psi}_{h}\right) \rightarrow \int_{0}^{T}(\mathbf{c} \otimes \underline{\mathbf{u}}, \nabla \boldsymbol{\psi})
$$

By the same arguments as, for instance, in [7] we get by $(3.37)_{2},(3.5),(3.39)$ that

$$
\int_{0}^{T}\left(\partial_{t} \mathbf{C}, \boldsymbol{\psi}_{h}\right)_{h} \rightarrow \int_{0}^{T}\left\langle\partial_{t} \mathbf{c}, \boldsymbol{\psi}\right\rangle
$$

The previous two identities and $(3.37)_{3},(3.39)$ yield in the limit

$$
\int_{0}^{T}\left\langle\partial_{t} \mathbf{c}, \boldsymbol{\psi}\right\rangle-\int_{0}^{T}(\mathbf{c} \otimes \underline{\mathbf{u}}, \nabla \boldsymbol{\psi})+\int_{0}^{T}(M \nabla \mathbf{w}, \nabla \boldsymbol{\psi})=0 .
$$

Using $(3.37)_{1,2},(3.37)_{3},(3.39)$ and (3.5) we easily verify that

$$
\begin{aligned}
\int_{0}^{T}\left(\nabla \mathbf{C}^{+}, \nabla \boldsymbol{\phi}_{h}\right) & \rightarrow \int_{0}^{T}(\nabla \mathbf{c}, \nabla \boldsymbol{\phi}), \\
\int_{0}^{T}\left[\left(A^{-} \mathbf{C}^{+}, \phi_{h}\right)_{h}+\left(A^{+} \mathbf{C}^{-}, \phi_{h}\right)_{h}\right] & \rightarrow \int_{0}^{T}(A \mathbf{c}, \boldsymbol{\phi}), \\
\int_{0}^{T}\left(\overline{\mathbf{W}}^{+}, \phi_{h}\right)_{h} & \rightarrow \int_{0}^{T}(\overline{\mathbf{w}}, \boldsymbol{\phi}) .
\end{aligned}
$$


Furthermore, we deduce from (3.10d), (3.36), (3.48) by the lower semi-continuity of norms that for all $\boldsymbol{\phi} \in C\left(0, T ; \mathbf{K} \cap \mathbf{H}^{2}(\Omega)\right)$

$$
\begin{aligned}
& \varepsilon \int_{0}^{T}(\nabla \mathbf{c}, \nabla \boldsymbol{\phi})-\varepsilon^{-1} \int_{0}^{T}(A \mathbf{c}, \boldsymbol{\phi}-\mathbf{c})-\int_{0}^{T}(\mathbf{w}, \boldsymbol{\phi}-\mathbf{c}) \\
& =\lim _{h, \tau \rightarrow 0} \varepsilon \int_{0}^{T}\left(\nabla \mathbf{C}^{+}, \nabla \boldsymbol{\phi}_{h}\right)-\varepsilon^{-1} \int_{0}^{T}\left[\left(A^{-} \mathbf{C}^{+}, \boldsymbol{\phi}_{h}-\mathbf{C}^{+}\right)_{h}+\left(A^{+} \mathbf{C}^{-}, \boldsymbol{\phi}_{h}-\mathbf{C}^{+}\right)_{h}\right] \\
& -\int_{0}^{T}\left(\mathbf{W}^{+}, \phi_{h}-\mathbf{C}^{+}\right)_{h} \\
& \geq \liminf _{h, \tau \rightarrow 0} \varepsilon \int_{0}^{T}\left\|\nabla \mathbf{C}^{+}\right\|^{2} \geq \varepsilon \int_{0}^{T}\|\nabla \mathbf{c}\|^{2} \geq 0
\end{aligned}
$$

where we use (3.32), i.e. the fact that the corresponding limits for $\overline{\mathbf{W}}^{+}$and $\mathbf{W}^{+}$coincide for $\phi \in C\left(0, T ; \mathbf{K} \cap \mathbf{H}^{2}(\Omega)\right)$.

Finally, we collect $(3.46),(3.47),(3.49)$ and conclude by a density argument that $(\underline{\mathbf{u}}, \mathbf{c}, \mathbf{w})$ satisfies (3.2).

\section{Numerical approximation of the density dependent model}

As discussed in Section 2, in order to derive an energy preserving numerical approximation of the density dependent model (1.1), it is convenient to consider the reformulation (2.7), (1.1b)-(1.1d). Hence, we propose the following fully discrete finite element approximation of the density dependent model (2.7), (1.1b)-(1.1d): For $k \geq 1$ find $\left(\underline{\mathbf{U}}^{k}, P^{k}, \mathbf{W}^{k}, \mathbf{C}^{k}\right) \in$ $\underline{\mathbf{W}}_{h} \times S_{h} \times \mathbf{S}_{h} \times \mathbf{S}_{h}^{+}$such that for all $(\underline{\mathbf{v}}, q, \boldsymbol{\psi}, \boldsymbol{\phi}) \in \underline{\mathbf{W}}_{h} \times S_{h} \times \mathbf{S}_{h} \times \mathbf{S}_{h}^{+}$the following holds

$$
\begin{aligned}
& \frac{1}{2}\left\{\left(\rho\left(\mathbf{C}^{k-1}\right) d_{t} \underline{\mathbf{U}}^{k}, \underline{\mathbf{v}}\right)+\left(d_{t}\left[\rho\left(\mathbf{C}^{k}\right) \underline{\mathbf{U}}^{k}\right], \underline{\mathbf{v}}\right)+\left(\left[\rho\left(\mathbf{C}^{k-1}\right) \underline{\mathbf{U}}^{k-1} \cdot \nabla\right] \underline{\mathbf{U}}^{k}, \underline{\mathbf{v}}\right)\right. \\
& \left.-\left(\left[\rho\left(\mathbf{C}^{k-1}\right) \underline{\mathbf{U}}^{k-1} \cdot \nabla\right] \underline{\mathbf{v}}, \underline{\mathbf{U}}^{k}\right)+\left(\left[\underline{\mathbf{J}}^{k} \cdot \nabla\right] \underline{\mathbf{U}}^{k}, \underline{\mathbf{v}}\right)-\left(\left[\underline{\mathbf{J}}^{k} \cdot \nabla\right] \underline{\mathbf{v}}, \underline{\mathbf{U}}^{k}\right)\right\} \\
& +\left(2 \mu\left(\mathbf{C}^{k-1}\right) D\left(\underline{\mathbf{U}}^{k}\right), D(\underline{\mathbf{v}})\right)+\left(P^{k}, \nabla \cdot \underline{\mathbf{v}}\right)=\left(\rho\left(\mathbf{C}^{k-1}\right) \underline{\mathbf{g}}, \underline{\mathbf{v}}\right)-\lambda\left(\left(\nabla \mathbf{W}^{k}\right)^{T} \mathbf{C}^{k-1}, \underline{\mathbf{v}}\right), \\
& \text { (4.1b) }\left(\nabla \cdot \underline{\mathbf{U}}^{k}, q\right)=0 \text {, } \\
& \text { (4.1c) }\left(d_{t} \mathbf{C}^{k}, \boldsymbol{\psi}\right)_{h}+\left(M \nabla \mathbf{W}^{k}, \nabla \boldsymbol{\psi}\right)=\left(\mathbf{C}^{k-1} \otimes \underline{\mathbf{U}}^{k}, \nabla \boldsymbol{\psi}\right), \\
& \text { (4.1d) } \varepsilon\left(\nabla \mathbf{C}^{k}, \nabla\left(\boldsymbol{\phi}-\mathbf{C}^{k}\right)\right)-\left(\varepsilon^{-1} A^{-} \mathbf{C}^{k}+\mathbf{W}^{k}, \boldsymbol{\phi}-\mathbf{C}^{k}\right)_{h} \geq \varepsilon^{-1}\left(A^{+} \mathbf{C}^{k-1}, \boldsymbol{\phi}-\mathbf{C}^{k}\right)_{h} \text {, }
\end{aligned}
$$

where $\underline{\mathbf{J}}^{k}=-\left(M \nabla \mathbf{W}^{k}\right)^{T} \boldsymbol{\rho}$ and $\mathbf{C}^{0}=\mathbf{Q}^{h} \mathbf{c}^{0} \in \mathbf{K}_{h}^{0}, \underline{\mathbf{U}}^{0}=\mathbf{P}_{0}^{h} \underline{\mathbf{u}}^{0} \in \underline{\mathbf{V}}_{h}$.

Lemma 5. The discrete solutions $\mathbf{C}^{k}, k=1, \ldots, K$ satisfy $\mathbf{C}^{k} \in \mathbf{K}_{h}^{0}$, i.e. in particular

$$
\int_{\Omega} \mathbf{C}^{k}=\int_{\Omega} \mathbf{C}^{0} \quad \text { and } \quad \mathbf{1}^{T} \mathbf{C}^{k}=1
$$


Furthermore, for $k=1, \ldots, K$ the solution $\left(\underline{\mathbf{U}}^{k}, \mathbf{C}^{k}, \mathbf{W}^{k}\right)$ of (4.1a-d) satisfies the energy estimate

$$
\begin{aligned}
\frac{\varepsilon \lambda}{2}\left\|\nabla \mathbf{C}^{k}\right\|^{2} & +\frac{1}{2}\left\|\rho^{\frac{1}{2}}\left(\mathbf{C}^{k}\right) \underline{\mathbf{U}}^{k}\right\|^{2}+\frac{\lambda}{\varepsilon}\left(\Psi\left(\mathbf{C}^{k}\right), 1\right)_{h} \\
+\tau \sum_{j=1}^{k}[ & \frac{\lambda}{2 \tau}\left\|\nabla\left(\mathbf{C}^{j}-\mathbf{C}^{j-1}\right)\right\|^{2} \\
& \left.+\frac{1}{2 \tau}\left\|\rho^{\frac{1}{2}}\left(\mathbf{C}^{j-1}\right)\left(\underline{\mathbf{U}}^{j}-\underline{\mathbf{U}}^{j-1}\right)\right\|^{2}+\lambda\left\|M^{\frac{1}{2}} \nabla \mathbf{W}^{j}\right\|^{2}+2\left\|\mu^{\frac{1}{2}}\left(\mathbf{C}^{j-1}\right) D\left(\underline{\mathbf{U}}^{j}\right)\right\|^{2}\right] \\
\leq & \tau \sum_{j=1}^{k}\left(\rho\left(\mathbf{C}^{j-1}\right) \underline{\mathbf{g}}, \underline{\mathbf{U}}^{j}\right)+\frac{\varepsilon \lambda}{2}\left\|\nabla \mathbf{C}^{0}\right\|^{2}+\frac{1}{2}\left\|\underline{\mathbf{U}}^{0}\right\|^{2}+\frac{\lambda}{\varepsilon}\left(\Psi\left(\mathbf{C}^{0}\right), 1\right)_{h} .
\end{aligned}
$$

Proof. The conservation properties (4.2) of $\mathbf{C}^{k}$ can be obtained in the same way as in Lemma 1. We set $\underline{\mathbf{v}}=\underline{\mathbf{U}}^{k}, q=P^{k}, \boldsymbol{\psi}=\mathbf{W}^{k}, \boldsymbol{\phi}=\mathbf{C}^{k-1}$ in (4.1a-d) and observe

$$
\begin{aligned}
\frac{1}{2}\left\{\left(\rho\left(\mathbf{C}^{k-1}\right) d_{t} \underline{\mathbf{U}}^{k}, \underline{\mathbf{U}}^{k}\right)+\left(d_{t}\left[\rho\left(\mathbf{C}^{k}\right) \underline{\mathbf{U}}^{k}\right], \underline{\mathbf{U}}^{k}\right)\right\} & \\
+2\left\|\mu^{\frac{1}{2}}\left(\mathbf{C}^{k-1}\right) D\left(\underline{\mathbf{U}}^{k}\right)\right\|^{2} & =\left(\rho\left(\mathbf{C}^{k-1}\right) \underline{\mathbf{g}}, \underline{\mathbf{v}}\right)-\lambda\left(\left(\nabla \mathbf{W}^{k}\right)^{T} \mathbf{C}^{k-1}, \underline{\mathbf{U}}^{k}\right), \\
\left(d_{t} \mathbf{C}^{k}, \mathbf{W}^{k}\right)_{h}+\left\|M^{\frac{1}{2}} \nabla \mathbf{W}^{k}\right\|^{2} & =\left(\mathbf{C}^{k-1} \otimes \underline{\mathbf{U}}^{k}, \nabla \mathbf{W}^{k}\right), \\
-\varepsilon\left(\nabla \mathbf{C}^{k}, \nabla d_{t} \mathbf{C}^{k}\right)+\left(\varepsilon^{-1} A^{-} \mathbf{C}^{k}+\mathbf{W}^{k}, d_{t} \mathbf{C}^{k}\right)_{h} & \geq-\varepsilon^{-1}\left(A^{+} \mathbf{C}^{k-1}, d_{t} \mathbf{C}^{k}\right)_{h} .
\end{aligned}
$$

We note the identity

$$
\begin{aligned}
\left(\rho\left(\mathbf{C}^{k-1}\right) d_{t} \underline{\mathbf{U}}^{k}, \underline{\mathbf{U}}^{k}\right)+\left(d_{t}\left[\rho\left(\mathbf{C}^{k}\right) \underline{\mathbf{U}}^{k}\right], \underline{\mathbf{U}}^{k}\right)= & \left\|\rho^{\frac{1}{2}}\left(\mathbf{C}^{k}\right) \underline{\mathbf{U}}^{k}\right\|^{2}+\left\|\rho^{\frac{1}{2}}\left(\mathbf{C}^{k-1}\right)\left(\underline{\mathbf{U}}^{k}-\underline{\mathbf{U}}^{k-1}\right)\right\|^{2} \\
& -\left\|\rho^{\frac{1}{2}}\left(\mathbf{C}^{k-1}\right) \underline{\mathbf{U}}^{k-1}\right\|^{2},
\end{aligned}
$$

multiply the last two equations in (4.3) by $\lambda$, sum up the resulting identities and obtain, similarly to (3.14), that

$$
\begin{aligned}
& \frac{1}{2}\left\{\left\|\rho^{\frac{1}{2}}\left(\mathbf{C}^{k}\right) \underline{\mathbf{U}}^{k}\right\|^{2}+\left\|\rho^{\frac{1}{2}}\left(\mathbf{C}^{k-1}\right)\left(\underline{\mathbf{U}}^{k}-\underline{\mathbf{U}}^{k-1}\right)\right\|^{2}-\left\|\rho^{\frac{1}{2}}\left(\mathbf{C}^{k-1}\right) \underline{\mathbf{U}}^{k-1}\right\|^{2}\right\} \\
& \quad+2\left\|\mu^{\frac{1}{2}}\left(\mathbf{C}^{k-1}\right) D\left(\underline{\mathbf{U}}^{k}\right)\right\|^{2}+\lambda\left\|M^{\frac{1}{2}} \nabla \mathbf{W}^{k}\right\|^{2} \\
& \quad+\lambda \varepsilon\left(\nabla \mathbf{C}^{k}, \nabla d_{t} \mathbf{C}^{k}\right)-\lambda \varepsilon^{-1}\left[\left(A^{-} \mathbf{C}^{k}, d_{t} \mathbf{C}^{k}\right)_{h}+\left(A^{+} \mathbf{C}^{k-1}, d_{t} \mathbf{C}^{k}\right)_{h}\right] \leq\left(\rho\left(\mathbf{C}^{k-1}\right) \underline{\mathbf{g}}, \underline{\mathbf{U}}^{k}\right) .
\end{aligned}
$$

The energy estimated then follows as in Lemma 2 after summing the above for $k$ replaced by $j=1, \ldots, k$. 


\section{NumericAl EXPERIMENTS}

Algorithm 1 can be generalized to the variable density nonlinear system (4.1a-d) by linearizing the additional nonlinear terms as

$$
\begin{aligned}
& \frac{1}{2}\left\{\left(\rho\left(\mathbf{C}^{k-1}\right) \frac{\underline{\mathbf{U}}^{k, l}-\underline{\mathbf{U}}^{k-1}}{\tau}, \underline{\mathbf{v}}\right)+\left(\frac{\rho\left(\mathbf{C}^{k, l}\right) \underline{\mathbf{U}}^{k, l}-\rho\left(\mathbf{C}^{k-1}\right) \underline{\mathbf{U}}^{k-1}}{\tau}, \underline{\mathbf{v}}\right)\right. \\
& \quad+\left(\left[\rho\left(\mathbf{C}^{k-1}\right) \underline{\mathbf{U}}^{k-1} \cdot \nabla\right] \underline{\mathbf{U}}^{k, l}, \underline{\mathbf{v}}\right)-\left(\left[\rho\left(\mathbf{C}^{k-1}\right) \underline{\mathbf{U}}^{k-1} \cdot \nabla\right] \underline{\mathbf{v}}, \underline{\mathbf{U}}^{k, l}\right)+\left(\left[\underline{\mathbf{J}}^{k, l} \cdot \nabla\right] \underline{\mathbf{U}}^{k, l}, \underline{\mathbf{v}}\right) \\
& \left.\quad-\left(\left[\underline{\mathbf{J}}^{k, l} \cdot \nabla\right] \underline{\mathbf{v}}, \underline{\mathbf{U}}^{k, l}\right)\right\}+\left(2 \mu\left(\mathbf{C}^{k-1}\right) D\left(\underline{\mathbf{U}}^{k, l}\right), D(\underline{\mathbf{v}})\right)+\left(P^{k, l}, \nabla \cdot \underline{\mathbf{v}}\right) \\
& \quad=\left(\rho\left(\mathbf{C}^{k-1}\right) \underline{\mathbf{g}}, \underline{\mathbf{v}}\right)-\lambda\left(\left(\nabla \mathbf{W}^{k, l}\right)^{T} \mathbf{C}^{k-1}, \underline{\mathbf{v}}\right), \\
& \left(\nabla \cdot \underline{\mathbf{U}}^{k, l}, q\right)=0, \\
& \left(\frac{\left.\mathbf{C}^{k, l}-\mathbf{C}^{k-1}, \boldsymbol{\psi}\right)_{h}+\left(M \nabla \mathbf{W}^{k, l}, \nabla \boldsymbol{\psi}\right)=\left(\mathbf{C}^{k-1} \otimes \underline{\mathbf{U}}^{k, l-1}, \nabla \boldsymbol{\psi}\right),}{\tau}\right. \\
& \varepsilon\left(\nabla \mathbf{C}^{k, l}, \nabla\left(\boldsymbol{\phi}-\mathbf{C}^{k, l}\right)\right)-\left(\varepsilon^{-1} A^{-} \mathbf{C}^{k, l}+\mathbf{W}^{k, l}, \boldsymbol{\phi}-\mathbf{C}^{k, l}\right)_{h} \geq \varepsilon^{-1}\left(A^{+} \mathbf{C}^{k-1}, \boldsymbol{\phi}-\mathbf{C}^{k, l}\right)_{h} .
\end{aligned}
$$

The tolerance for the termination of the fixed-point algorithm is chosen $\delta_{f i x}=10^{-8}$ and the algorithm typically terminated within 3-6 iterations for sufficiently small time step sizes.

In practice we see very little difference between the numerical results obtained by the fixedpoint algorithm and by the semi-implicit linear scheme

$$
\begin{aligned}
& \frac{1}{2}\left\{\left(\rho\left(\mathbf{C}^{k-1}\right) d_{t} \underline{\mathbf{U}}^{k}, \underline{\mathbf{v}}\right)+\left(d_{t}\left[\rho\left(\mathbf{C}^{k}\right) \underline{\mathbf{U}}^{k}\right], \underline{\mathbf{v}}\right)\right. \\
& \quad+\left(\left[\rho\left(\mathbf{C}^{k-1}\right) \underline{\mathbf{U}}^{k-1} \cdot \nabla\right] \underline{\mathbf{U}}^{k}, \underline{\mathbf{v}}\right)-\left(\left[\rho\left(\mathbf{C}^{k-1}\right) \underline{\mathbf{U}}^{k-1} \cdot \nabla\right] \underline{\mathbf{v}}, \underline{\mathbf{U}}^{k}\right)+\left(\left[\underline{\mathbf{J}}^{k} \cdot \nabla\right] \underline{\mathbf{U}}^{k}, \underline{\mathbf{v}}\right) \\
& \left.\quad-\left(\left[\underline{\mathbf{J}}^{k} \cdot \nabla\right] \underline{\mathbf{v}}, \underline{\mathbf{U}}^{k}\right)\right\}+\left(2 \mu\left(\mathbf{C}^{k-1}\right) D\left(\underline{\mathbf{U}}^{k}\right), D(\underline{\mathbf{v}})\right)+\left(P^{k}, \nabla \cdot \underline{\mathbf{v}}\right) \\
& \quad=\left(\rho\left(\mathbf{C}^{k-1}\right) \underline{\mathbf{g}}, \underline{\mathbf{v}}\right)-\lambda\left(\left(\nabla \mathbf{W}^{k}\right)^{T} \mathbf{C}^{k-1}, \underline{\mathbf{v}}\right), \\
& \left(\nabla \cdot \underline{\mathbf{U}}^{k}, q\right)=0, \\
& \left(d_{t} \mathbf{C}^{k}, \boldsymbol{\psi}\right)_{h}+\left(M \nabla \mathbf{W}^{k}, \nabla \boldsymbol{\psi}\right)=\left(\mathbf{C}^{k-1} \otimes \underline{\mathbf{U}}^{k-1}, \nabla \boldsymbol{\psi}\right), \\
& \varepsilon\left(\nabla \mathbf{C}^{k}, \nabla\left(\boldsymbol{\phi}-\mathbf{C}^{k}\right)\right)-\left(\varepsilon^{-1} A^{-} \mathbf{C}^{k}+\mathbf{W}^{k}, \boldsymbol{\phi}-\mathbf{C}^{k}\right)_{h} \geq \varepsilon^{-1}\left(A^{+} \mathbf{C}^{k-1}, \boldsymbol{\phi}-\mathbf{C}^{k}\right)_{h},
\end{aligned}
$$

which is the same as (4.1a-d), but with $\underline{\mathbf{U}}^{k}$ in (4.1c) replaced by $\underline{\mathbf{U}}^{k-1}$, i.e., the scheme above is equivalent to performing only one iteration of the adapted Algorithm 1. For the linear scheme (5.2a-d) the energy decrease is not guaranteed, however, in the computations below, the energy graphs for both schemes were graphically indistinguishable. Hence, the computationally more demanding simulations for $N=5$ components were performed using the decoupled linear scheme $(5.2 \mathrm{a}-\mathrm{d})$.

The advantage of the fixed-point Algorithm 1, as well as of the semi-implicit scheme (5.2a-d), is that in each iteration we first solve the Cahn-Hilliard variational inequality $(3.15 \mathrm{c})-(3.15 \mathrm{~d})$ and then the Navier-Stokes part (3.15a)-(3.15b) in a decoupled manner. The Navier-Stokes part corresponds to a linear system of equations and is solved using the direct solver UMFPACK, see [12]. The Cahn-Hilliard part is a linear variational inequality and is solved using a projected block Gauss-Seidel algorithm from [29], with a tolerance taken between $10^{-6}$ in experiments with $N=5$, and $10^{-8}$ otherwise. 
In the case of equal surface tension coefficients (1.8), we set $A^{-}=-\frac{N-1}{N} \mathbf{1 1}^{T}$, while in the case of variable surface tension we choose $A^{+}=\operatorname{diag}_{i}\left\{\sum_{j=1, j \neq i}^{N}\left|A_{i j}\right|\right\}$.

To increase computational efficiency we employ an adaptive algorithm, which enforces a finer mesh size $h \equiv h_{f}=1 / N_{f}$ within the diffuse interfacial region and a coarser mesh size $h \equiv$ $h_{c}=1 / N_{c}$ otherwise. The diffuse interfacial region is defined as $\left\{\underline{\mathbf{x}} \in \Omega ; \operatorname{dist}\left\{\mathbf{c}(\underline{\mathbf{x}}), \partial \mathbb{G}^{N}\right\}>\right.$ $\left.\delta_{t o l}\right\}$, typically $\delta_{t o l} \approx 10^{-8}$, see [29] for a more detailed description. Of course, we note that the mesh still has to be sufficiently fine outside of the interfacial region in order to obtain sufficiently accurate approximations of the Navier-Stokes part of the system. Typically we chose $N_{f}=128, N_{c}=N_{f} / 4$ and $\varepsilon=(16 \pi)^{-1}$.

5.1. Static contact angles. We examine the stationary contact angles for 3-component flow with constant density $\rho_{i}=1$ and viscosity $\mu_{i}=0.1, i=1, \ldots, N$. An in-depth study without the coupling to the Navier-Stokes equations has been performed in [29]. The considered fluid configuration is displayed in Figure 1. According to Young's law, stationary contact angles satisfy

$$
\frac{\sin \theta_{1}}{\sigma_{23}}=\frac{\sin \theta_{2}}{\sigma_{13}}=\frac{\sin \theta_{3}}{\sigma_{12}},
$$

where $\sigma_{i j}$ is the surface tension coefficient between the $i$-th and $j$-th fluid phases, and $\theta_{k}$ is the angle at the triple junction inside the $k$-th phase. We consider four different choice of the

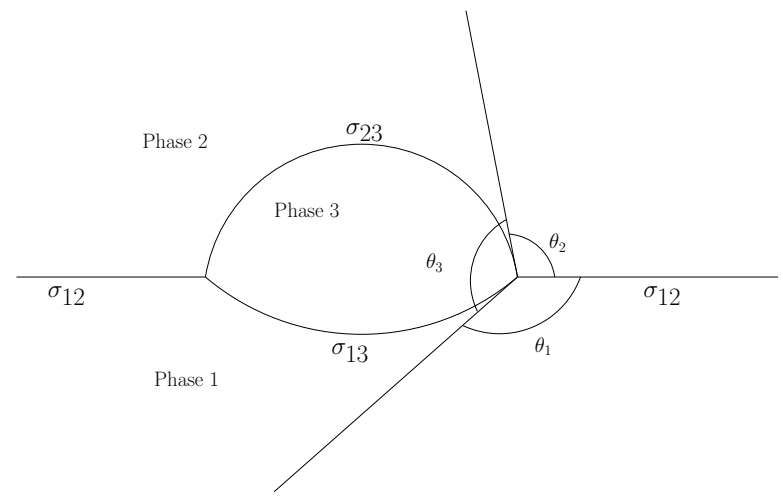

FiguRE 1. Contact angles predicted by Young's law.

surface tension matrix:
a) $A=\left(\begin{array}{rrr}0 & -1 & -1 \\ -1 & 0 & -1 \\ -1 & -1 & 0\end{array}\right)$
b) $A=\left(\begin{array}{rrr}0 & -2 & -1 \\ -2 & 0 & -1 \\ -1 & -1 & 0\end{array}\right)$,

and
c) $A=\frac{1}{2}\left(\begin{array}{rrr}0 & -1 & -2 \\ -1 & 0 & -2 \\ -2 & -2 & 0\end{array}\right)$
d) $A=\frac{1}{2}\left(\begin{array}{rrr}0 & -2 & -1 \\ -2 & 0 & -2 \\ -1 & -2 & 0\end{array}\right)$.

As discussed in Section 2.4 we have $\left|A_{i j}\right| \approx \sigma_{i j}^{2}$ and Young's law (5.3) implies the following contact angles: a) $\theta_{1}=\theta_{2}=\theta_{3} \approx \frac{2}{3} \pi=120^{\circ}$; b) $\theta_{3} \approx 2 \arccos \left(\frac{1}{2} \sqrt{\frac{A_{12}}{A_{13}}}\right) \approx 90^{\circ}$ and $\left.\theta_{1}=\theta_{2} ; c\right)$ $\theta_{3} \approx 2 \arccos \left(\frac{1}{2} \sqrt{\frac{A_{12}}{A_{13}}}\right) \approx 138.6^{\circ}$ and $\left.\theta_{1}=\theta_{2} ; d\right) \theta_{2} \approx 2 \arccos \left(\frac{1}{2} \sqrt{\frac{A_{13}}{A_{12}}}\right) \approx 138.6^{\circ}$ and $\theta_{1}=\theta_{3}$. 
We employ zero initial condition for the velocity, constant mobility $(1.11)$, set $\lambda=10^{-3}$, $\varepsilon=(16 \pi)^{-1}, m_{0}=10^{-5}, \tau=2 \times 10^{-3}, \mathbf{g}=\underline{\mathbf{0}}$ and compute until a steady state is reached. We display the results in Figure 2, where we visualize the interface by displaying the function $c_{\text {int }}=\left(1-c_{1}\right)\left(1-c_{2}\right)\left(1-c_{3}\right)$ which is nonzero only within the diffuse interface. We observe that at least qualitatively the results agree with Young's law.
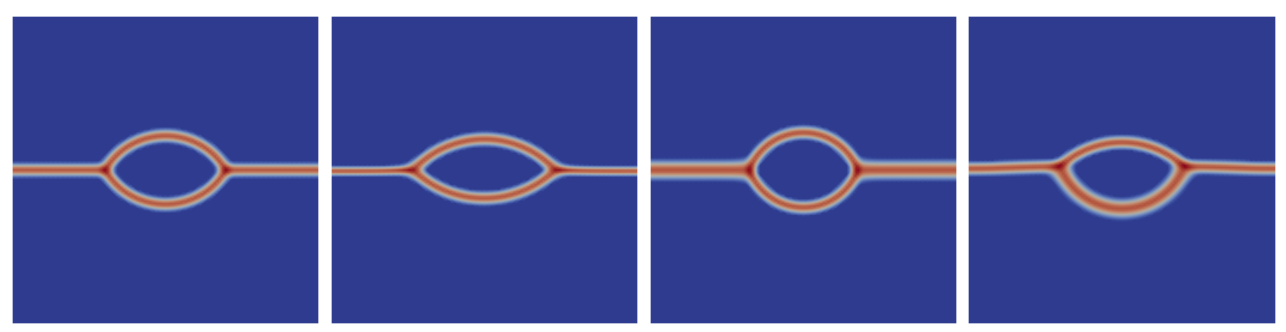

FiguRE 2. Solution $c_{i n t}$ for cases $a$ )-d) (from left to right).

5.2. Surface tension effects. We present a computational study to demonstrate the effect of different surface tension parameters. We consider a mixture of five fluids, i.e. $N=5$, with equal densities and viscosities $\rho_{i}=1, i=1, \ldots, 5, \mu_{i}=1$ and set $\mathbf{g}=\underline{\mathbf{0}}$. We consider the domain $\Omega=(0,1) \times(0,5)$ and prescribe homogeneous Dirichlet boundary conditions for the velocity field and homogeneous Neumann boundary conditions for $\mathbf{c}, \mathbf{w}$. The initial condition for the velocity is $\underline{\mathbf{u}}^{0}=\underline{\mathbf{0}}$. The initial condition for $\mathbf{c}^{0}$ is close to the stationary profile displayed in Figure 3 (left); the four circular bubbles with radius 0.25 from bottom to top contain fluids two to five, respectively, and the remaining part of the domain is occupied by fluid one.

In the computations we use the concentration dependent mobility (1.12) with $m_{0}=10^{-5}$ and $\nu=10^{-8}$ and the matrix $A$ is taken as

$$
A=\frac{1}{16}\left(\begin{array}{rrrrr}
0 & -32 & -8 & -2 & -1 \\
-32 & 0 & -16 & -16 & -16 \\
-8 & -16 & 0 & -16 & -16 \\
-2 & -16 & -16 & 0 & -16 \\
-1 & -16 & -16 & -16 & 0
\end{array}\right)
$$

As discussed in Section 2.4, the choice of coefficients of $A$ implies that the surface tension coefficients $\sigma_{i j}$ between the $j$-th and $i$-th fluid satisfy: $\sigma_{12} / \sigma_{13} \approx \sqrt{A_{12} / A_{13}}=2, \sigma_{13} / \sigma_{14} \approx$ $\sqrt{A_{13} / A_{14}}=2, \sigma_{14} / \sigma_{15} \approx \sqrt{A_{14} / A_{15}}=\sqrt{2}$ and $\sigma_{i j} / \sigma_{k l}=1$, otherwise.

The remaining parameters were chosen $\lambda=0.1, \varepsilon=(16 \pi)^{-1}, \tau=2 \times 10^{-3}, h_{\text {min }}=1 / 128$, $h_{\max }=\frac{1}{4}$.

The pressure distribution is displayed in Figure 3. In order to eliminate the effect of the pressure differences within the interfaces and highlight the distribution of the pressure within the pure phases we also display in Figure 3 a normalized pressure where we set the pressure within interfaces equal to the pressure at the reference point $\underline{\mathbf{x}}_{0}=(0.25,0.25)$.

According to the Young-Laplace law the pressure difference across the fluid interface $\Delta_{i j} p=$ $\sigma_{i j} \kappa_{i j}$ where $\kappa_{i j}$ is the curvature of the interface between fluids $i$ and $j$. Since the interfaces in the computed solution are circular we have that $\kappa_{i j} \approx-4$ for $i=1, j=2, \ldots, 5$. To estimate $\Delta_{i j} p$ we measure the difference between the pressure in the center of each bubble and the pressure at the reference point $\underline{\mathbf{x}}_{0}$. We obtain the pressure differences are $\Delta_{12} p=-0.461954$, $\Delta_{13} p-0.221757, \Delta_{14} p=-0.11103, \Delta_{14} p=-0.0788834$. The results are in good agreement 
with the theoretical predictions. The larger difference for the value of $\Delta_{12} p$ can be explained by the fact that the mesh size has not been sufficiently fine to properly approximate the interface, cf. Figure 3 (right).
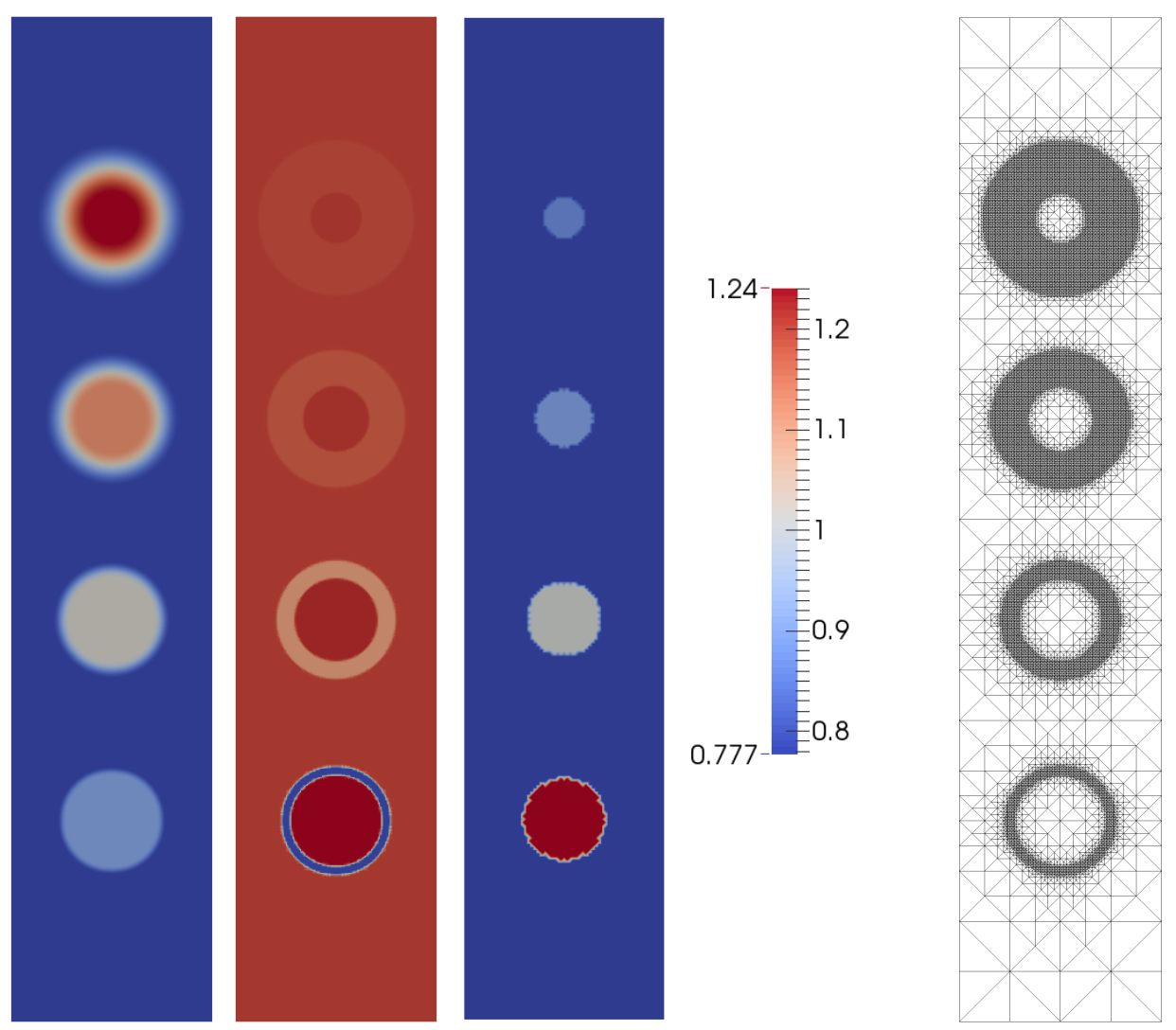

FiguRE 3. Solution at final time, computed pressure, normalized pressure and the finite element mesh (from left to right).

5.3. 3-component flow with variable density. We consider a mixture of three fluids with densities $\rho_{i}=4-i, i=1, \ldots, 3$, in a gravitational field. The initial condition for the density is displayed in Figure 4. The heavier fluids are positioned above the lighter ones, the initial interfaces have a sinusoidal shape and all three fluid components have equal mass. The initial condition for the velocity is $\underline{\mathbf{u}}^{0}=\underline{\mathbf{0}}$. We consider homogeneous Dirichlet boundary conditions for the velocity field and homogeneous Neumann boundary conditions for $\mathbf{c}, \mathbf{w}$. We choose $\underline{\mathbf{g}}=(0,-1)^{T}, \mu_{i}=10^{-2}, i=1, \ldots, 3, \lambda=10^{-3}, \tau=2 \times 10^{-3}, h_{\min }=1 / 128, h_{\max }=1 / 16$ and employ the constant mobility (1.11) with $m_{0}=10^{-5}$.

The evolution of the density is displayed in Figure 4. Due to the perturbation of the interface the fluids form the so-called Rayleigh-Taylor instability. In Figure 5 we display results for the same problem computed with the density dependent model with $\underline{\mathbf{j}}=\underline{\mathbf{0}}$, which is a formulation that is most frequently used in the literature. The numerical approximation of this model corresponds to (3.10a-d) with $\underline{\mathbf{J}}^{k}=\underline{\mathbf{0}}$ for $k=0, \ldots, K$. There are obvious differences between the solutions for the two models but qualitatively, the results are comparable. 

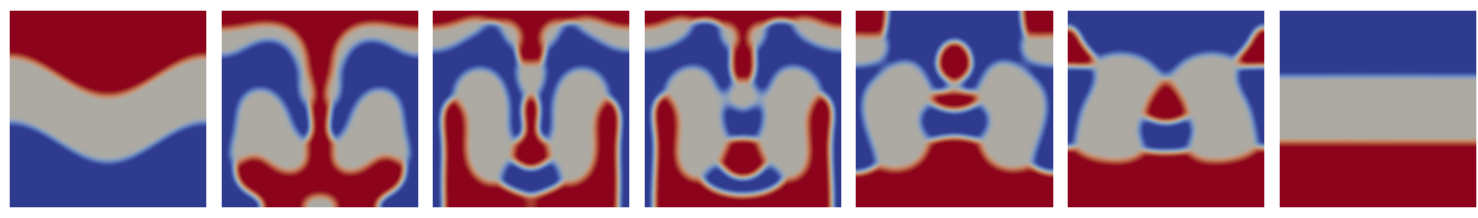

Figure 4. Evolution of the density of a three component mixture at $t=$ $0,4,5.4,5.8,9,11.6$ (from left to right).
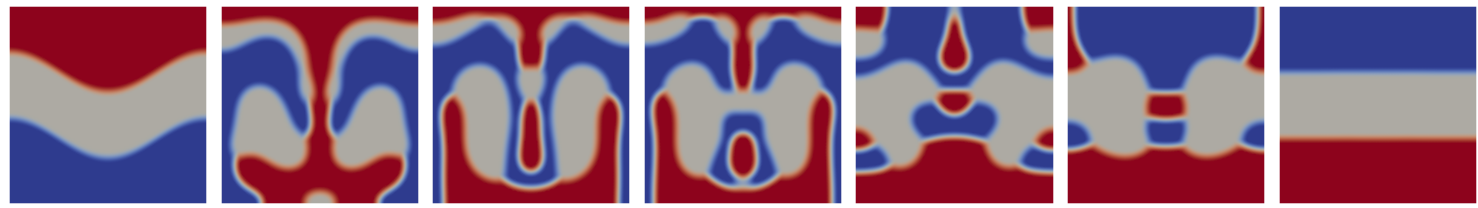

Figure 5. Evolution of the density of a three component mixture at $t=$ 0, 4, 5.4, 5.8, 9, 11.6 (from left to right), CHNS model with $\underline{\mathbf{j}}=\underline{\mathbf{0}}$.

5.4. 5-component flow. We consider a mixture of five fluids with densities $\rho_{i}=6-i$, $i=1, \ldots, 5$, in a gravitational field. The initial condition for the density is displayed in Figure 6 . The heavier fluids are positioned above the lighter ones, the initial interfaces have a sinusoidal shape and all five fluid components have equal mass. The initial condition for the velocity is $\underline{\mathbf{u}}^{0}=\underline{\mathbf{0}}$. We consider homogeneous Dirichlet boundary conditions for the velocity field and homogeneous Neumann boundary conditions for $\mathbf{c}, \mathbf{w}$. We choose $\mathbf{g}=(0,-1)^{T}$, $\mu_{i}=3.3 \times 10^{-4}, i=1, \ldots, 5, \lambda=0, \tau=2 \times 10^{-3}, h_{\min }=1 / 128, h_{\max }=1 / 16$ and employ the constant mobility (1.11) with $m_{0}=10^{-5}$.

The evolution of the density is displayed in Figure 6. Due to the perturbation of the interface the fluids form the so-called Rayleigh-Taylor instability. We obtain similar results as [26], however they appear to use periodic boundary conditions on the left and right hand side boundaries, so the results are not directly comparable.

For comparison, in Figure 7 we also display results computed with $\mathbf{j}=\underline{\mathbf{0}}$. The differences between the solutions for the two different models become increasingly significant as the geometry of the interfaces becomes more complex (note that the term $\underline{\mathbf{j}}$ acts only within the interfacial regions).

We repeat the calculation for the model with concentration dependent mobility (1.12) with $\delta=10^{-18}, m_{0}=3.2^{-4}$ and $\lambda=10^{-3}$; the remaining parameters remain the same as above. The evolution of the density is displayed in Figure 8. The evolution of the total energy (cf. $(2.4))$

$$
\mathcal{E}_{t o t}=\lambda \mathcal{E}_{c h}(\mathbf{c})+\mathcal{E}_{n s}(\mathbf{c}, \underline{\mathbf{u}})+\int_{0}^{t} \int_{\Omega} 2 \mu(\mathbf{c})|D(\underline{\mathbf{u}})|^{2}-\int_{0}^{t} \int_{\Omega} \rho(\mathbf{c}) \underline{\mathbf{g}} \cdot \underline{\mathbf{u}},
$$

in Figure 9 demonstrates that the physically relevant energy of the computed numerical approximation is decreasing in time.

\section{REFERENCES}

[1] Helmut Abels, Harald Garcke, and Günther Grün. Thermodynamically consistent, frame indifferent diffuse interface models for incompressible two-phase flows with different densities. Math. Models Methods Appl. Sci., 22(3):1150013, 2012. 

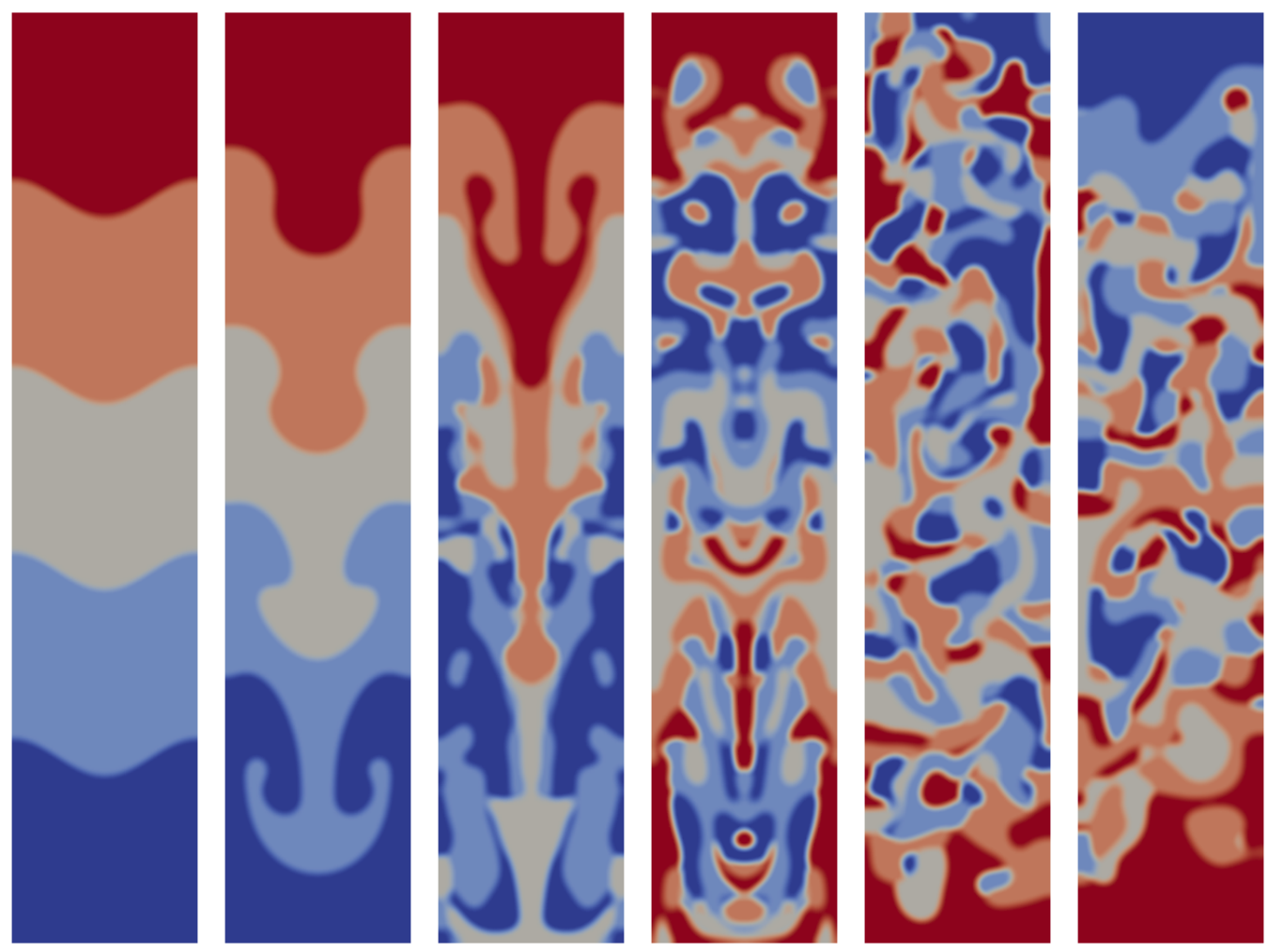

Figure 6. Evolution of the density of a five component mixture at $t=$ 0,3,6,10,20, 40 (from left to right).

[2] L'ubomír Baňas and Andreas Prohl. Convergent finite element discretization of the multi-fluid nonstationary incompressible magnetohydrodynamics equations. Math. Comp., 79(272):1957-1999, 2010.

[3] John W. Barrett and James F. Blowey. An error bound for the finite element approximation of a model for phase separation of a multi-component alloy. IMA J. Numer. Anal., 16:257-287, 1996.

[4] John W. Barrett and James F. Blowey. Finite element approximation of a model for phase separation of a multi-component alloy with non-smooth free energy. Numer. Math., 77(1):1-34, 1997.

[5] John W. Barrett and James F. Blowey. Finite element approximation of a model for phase separation of a multi-component alloy with a concentration-dependent mobility matrix. IMA J. Numer. Anal., 18(2):287328, 1998.

[6] John W. Barrett and James F. Blowey. Finite element approximation of a model for phase separation of a multi-component alloy with nonsmooth free energy and a concentration dependent mobility matrix. Math. Models Methods Appl. Sci., 9(5):627-663, 1999.

[7] John W. Barrett, James F. Blowey, and Harald Garcke. On fully practical finite element approximations of degenerate Cahn-Hilliard systems. M2AN Math. Model. Numer. Anal., 35(4):713-748, 2001.

[8] James F. Blowey, M. I. M. Copetti, and Charles M. Elliott. The numerical analysis of a model for phase separation of a multi-component alloy. IMA J. of Numer. Anal., 16:111-139, 1996.

[9] Franck Boyer and Céline Lapuerta. Study of a three component Cahn-Hilliard flow model. M2AN Math. Model. Numer. Anal., 40(4):653-687, 2006.

[10] Franck Boyer and Sebastian Minjeaud. Numerical schemes for a three component Cahn-Hilliard model. M2AN Math. Model. Numer. Anal., 45(4):697-738, 2011.

[11] Franck Boyer and Sebastian Minjeaud. Hierarchy of consistent n-component Cahn-Hilliard systems. Math. Models Methods Appl. Sci., 24(14):2885-2928, 2014.

[12] Timothy A. Davis. Algorithm 832: UMFPACK V4.3 - an unsymmetric-pattern multifrontal method. ACM Trans. Math. Software, 30(2):196-199, 2004. 

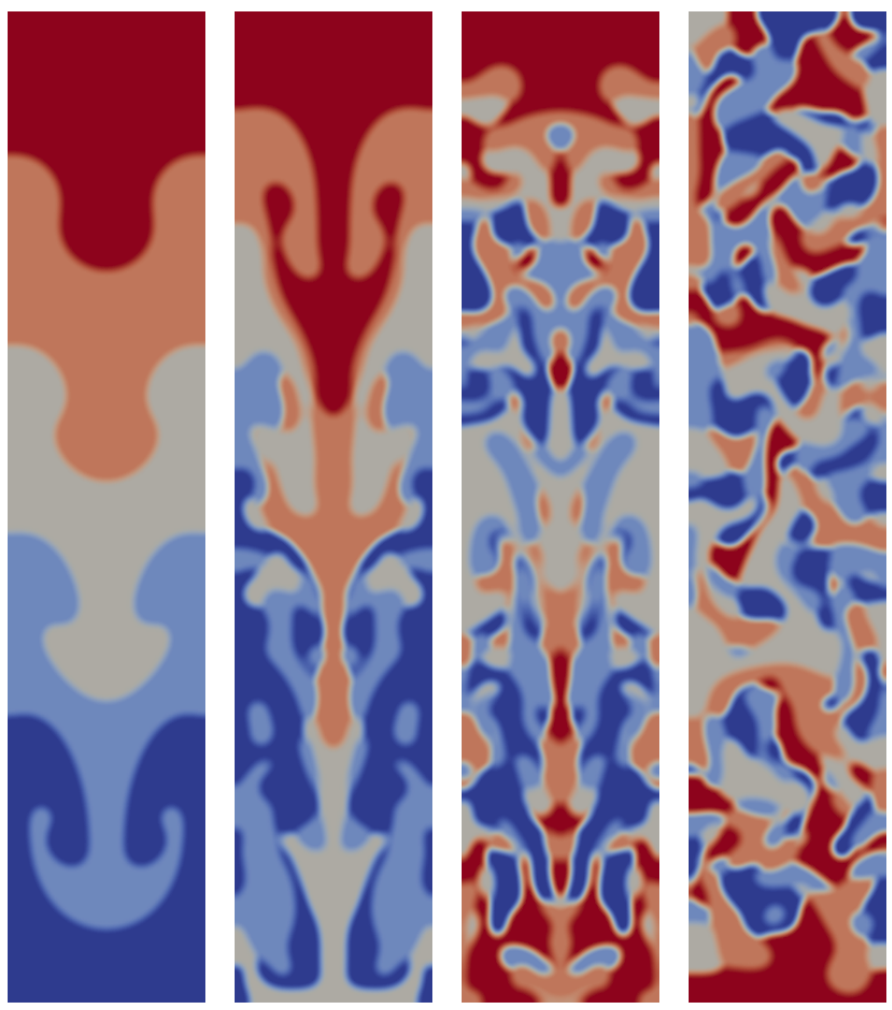

Figure 7. Evolution of the density of a five component mixture at $t=$ $3,6,10,20$ computed with $\underline{\mathbf{j}}=\underline{\mathbf{0}}$ (from left to right).

[13] S. Dong. An efficient algorithm for incompressible N-phase flows. J. Comput. Phys., 276:691-728, 2014.

[14] S. Dong. Physical formulation and numerical algorithm for simulating $\mathrm{N}$ immiscible incompressible fluids involving general order parameters. J. Comput. Phys., 283:98-128, 2015.

[15] Charles M. Elliott and H. Garcke. Diffusional phase transitions in multicomponent systems with a concentration dependent mobility matrix. Phys. D, 109:242-256, 1997.

[16] Xiaobing Feng. Fully discrete finite element approximations of the Navier-Stokes-Cahn-Hilliard diffuse interface model for two-phase fluid flows. SIAM J. Numer. Anal., 44(3):1049-1072, 2006.

[17] Harald Garcke, Michael Hinze, and Christian Kahle. A stable and linear time discretization for a thermodynamically consistent model for two-phase incompressible flow. Appl. Numer. Math., 99:151-171, 2016.

[18] G. Grün. On convergent schemes for diffuse interface models for two-phase flow of incompressible fluids with general mass densities. SIAM J. Numer. Anal., 51(6):3036-3061, 2013.

[19] Günther Grün, Francisco Guillén-González, and Stefan Metzger. On Fully Decoupled, Convergent Schemes for Diffuse Interface Models for Two-Phase Flow with General Mass Densities. Commun. Comput. Phys., 19(5):1473-1502, 2016.

[20] G. Grün and F. Klingbeil. Two-phase flow with mass density contrast: Stable schemes for a thermodynamic consistent and frame-indifferent diffuse-interface model. J. Comput. Phys., 257:708-725, 2014.

[21] David Kay, Vanessa Styles, and Richard Welford. Finite element approximation of a Cahn-HilliardNavier-Stokes system. Interfaces Free Bound., 10(1):15-43, 2008.

[22] Junseok Kim. A continuous surface tension force formulation for diffuse-interface models. J. Comput. Phys., 204(2):784-804, 2005.

[23] Junseok Kim. A generalized continuous surface tension force formulation for phase-field models for multicomponent immiscible fluid flows. Comput. Methods Appl. Mech. Engrg., 198(37-40):3105-3112, 2009.

[24] Junseok Kim. Phase-field models for multi-component fluid flows. Commun. Comput. Phys., 12(3):613$661,2012$. 

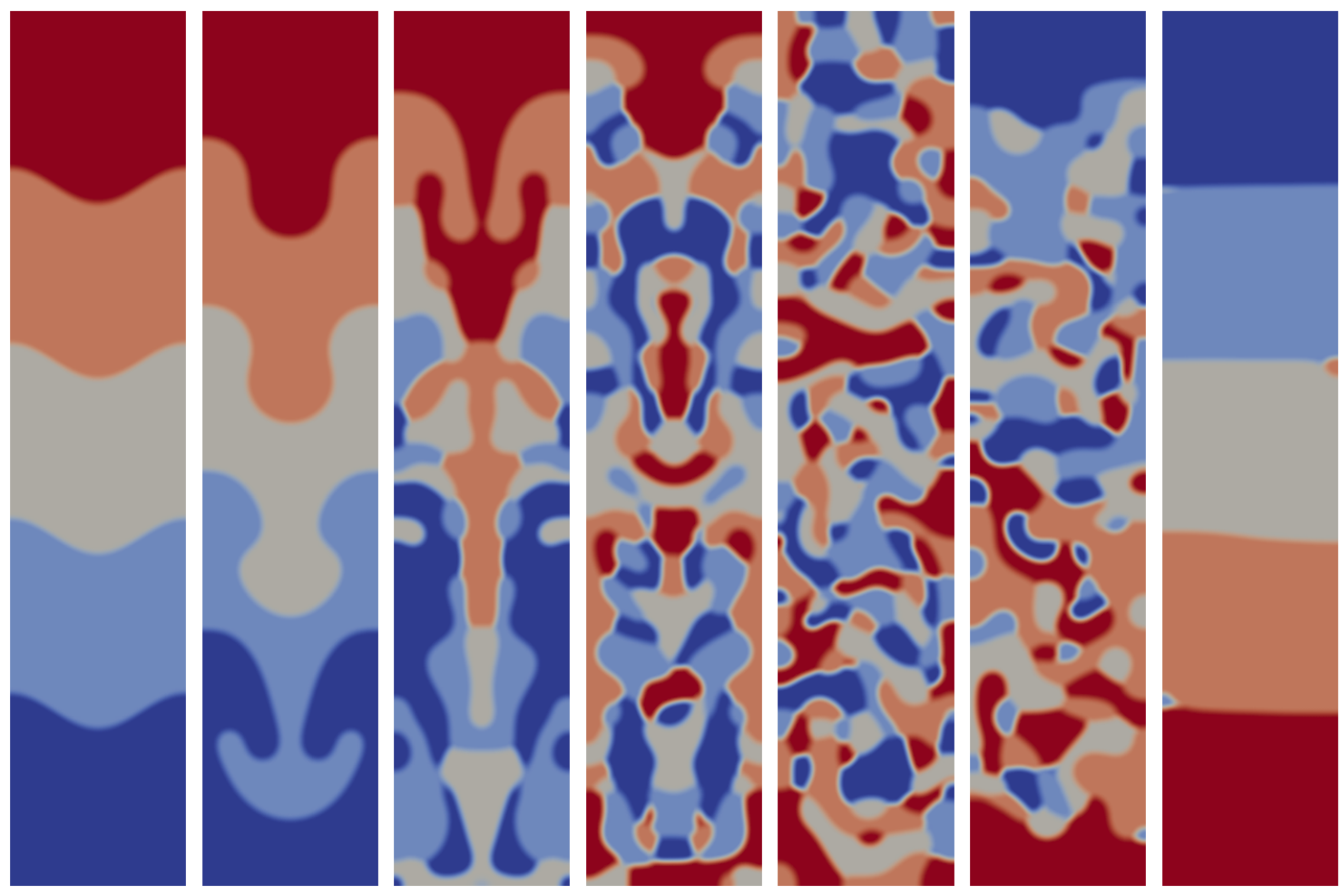

FiguRE 8. Evolution of the density of a five component mixture with concentration dependent mobility at $t=0,3,6,10,20,40,200$ (from left to right).

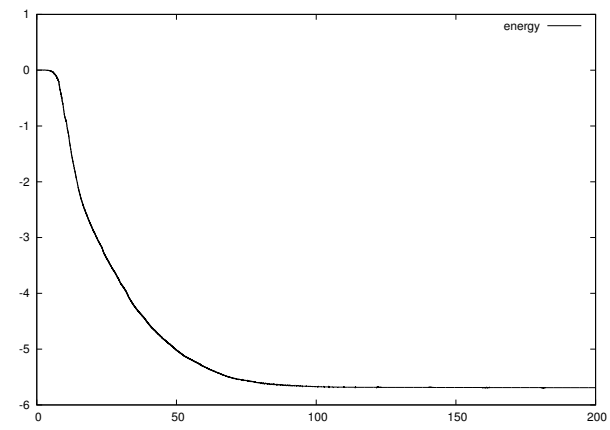

Figure 9. Evolution of the total energy of a five component mixture with concentration dependent mobility.

[25] Junseok Kim and John Lowengrub. Phase field modeling and simulation of three-phase flows. Interfaces Free Bound., 7(4):435-466, 2005.

[26] Hyun Geun Lee, Jeong-Whan Choi, and Junseok Kim. A practically unconditionally gradient stable scheme for the N-component Cahn-Hilliard system. Physica A, 391(4):1009-1019, 2012.

[27] Chun Liu and Noel J. Walkington. Convergence of numerical approximations of the incompressible NavierStokes equations with variable density and viscosity. SIAM J. Numer. Anal., 45(3):1287-1304, 2007.

[28] Sebastian Minjeaud. An unconditionally stable uncoupled scheme for a triphasic Cahn-Hilliard/NavierStokes model. Numer. Methods Partial Differential Equations, 29(2):584-618, 2013. 
[29] Robert Nürnberg. Numerical simulations of immiscible fluid clusters. Appl. Numer. Math., 59(7):16121628, 2009.

[30] Roger Temam. Navier-Stokes Equations, volume 2 of Studies in Mathematics and its Applications. NorthHolland Publishing Co., Amsterdam, third edition, 1984.

Department of Mathematics, Bielefeld University, 33501 Bielefeld, Germany

E-mail address: banas@math.uni-bielefeld.de

Department of Mathematics, Imperial College London, London, SW7 2AZ, UK

E-mail address: robert.nurnberg@imperial.ac.uk 